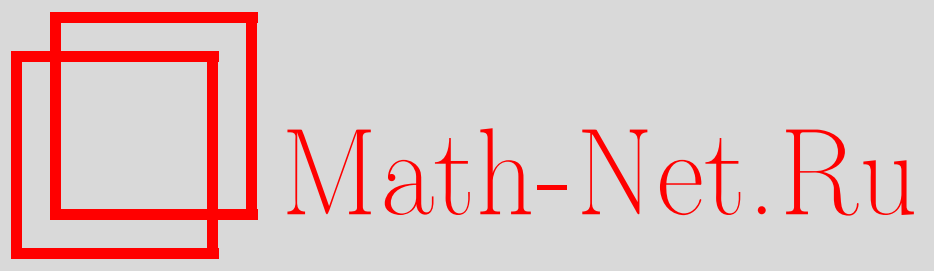

Г. Г. Граховски, Д. И. Мустафа, Х. Сусанто, О нелокальных редукциях многокомпонентного нелинейного уравнения Шредингера в симметрических пространствах, TMФ, 2018, том 197, номер 1, 45-67

DOI: https://doi.org/10.4213/tmf9507

Использование Общероссийского математического портала Math-Net.Ru подразумевает, что вы прочитали и согласны с пользовательским соглашением http://www . mathnet.ru/rus/agreement

Параметры загрузки:

IP : 3.81 .55 .215

26 апреля 2023 г., 17:19:30

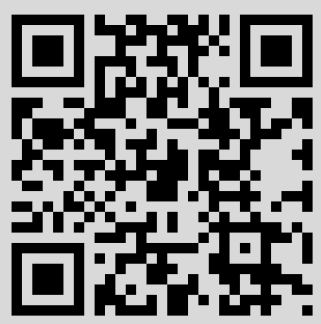




\section{О НЕЛОКАЛЬНЫХ РЕДУКЦИЯХ МНОГОКОМПОНЕНТНОГО НЕЛИНЕЙНОГО УРАВНЕНИЯ ШРЕДИНГЕРА В СИММЕТРИЧЕСКИХ ПРОСТРАНСТВАХ}

Цель данной работы - построить обратное преобразование рассеяния для многокомпонентных обобщений нелокальных редукций нелинейного уравнения Шредингера, обладающего $\mathcal{P} \mathcal{T}$-симметрией относительно симметрических пространств, т.е. получить спектральные свойства ассоциированного оператора Лакса, функцию Йоста, матрицу рассеяния и минимальное множество данных рассеяния, фундаментальные аналитические решения. В качестве основных примеров рассматриваются векторное обобщение Манакова для уравнения Шредингера (связанное с симметрическими пространствами типа A.III) и многокомпонентные нелинейные уравнения Шредингера типа Кулиша-Склянина (связанные с симметрическими пространствами типа BD.I). Кроме того, однои двухсолитонные решения получены с помощью подходящей модификации метода одевания Захарова-Шабата. Показано, что многокомпонентные нелинейные уравнения Шредингера этого типа допускают регулярные и сингулярные солитонные конфигурации. Наконец, представлены различные примеры однои двухсолитонных решений для моделей обоих типов, допускающих различные редукции.

Ключевые слова: интегрируемая система, многокомпонентные нелинейные уравнения Шредингера, представление Лакса, система Захарова-Шабата, спектральные разложения, $\mathcal{P} \mathcal{T}$-симметрия, обратное преобразование рассеяния, задача Римана-Гильберта, метод одевания.

DOI: https://doi.org/10.4213/tmf9507

\section{1. ВВЕДЕНИЕ}

Одним из наиболее важных и наиболее часто встречающихся вполне интегрируемых нелинейных уравнений в частных производных является нелинейное уравнение Шредингера (НУШ) [1]-[4]

$$
i q_{t}+q_{x x}+2|q|^{2} q(x, t)=0
$$

${ }^{*}$ Department of Mathematical Sciences, University of Essex, Colchester, UK.

E-mail: grah@essex.ac.uk, jimust@essex.ac.uk, hsusanto@essex.ac.uk 
Здесь $q(x, t)$ - комплекснозначная функция, достаточно быстро стремящаяся к нулю при $|x| \rightarrow \infty$ [4], [5]. Это уравнение было получено как основное уравнение, описывающее процессы и явления в таких разных областях физики, как глубоководные волны, физика плазмы, нелинейная волоконная оптика. Например, в оптике НУШ моделирует распространение волн в керровских средах, где нелинейность пропорциональна интенсивности поля.

НУШ (1) возникло на раннем этапе развития метода обратной задачи рассеяния (МОЗР) и теории солитонов [2]-[7] и обладает всеми замечательными свойствами уравнений в частных производных и систем уравнений в частных производных, интегрируемых с помощью МОЗР, а именно допускает солитонные решения, бесконечное множество интегралов движения, мультигамильтонову формулировку и т. д. [2], [4]. Основным инструментом изучения интегрируемости с помощью МОЗР является существование представления Лакса для нелинейного эволюционного уравнения (НЭУ), т. е. НЭУ можно представить как условие совместности двух линейных операторов [1]-[4], [8]. Задача рассеяния для уравнения (1) задается системой Захарова-Шабата (связанной с алгеброй $\operatorname{sl}(2, \mathbb{C})$ ):

$$
\begin{aligned}
& L \chi \equiv\left(i \frac{d}{d x}+q(x, t)-\lambda \sigma_{3}\right) \chi(x, t, \lambda)=0, \\
& q(x, t)=\left(\begin{array}{cc}
0 & q^{+} \\
q^{-} & 0
\end{array}\right), \quad \sigma_{3}=\left(\begin{array}{cc}
1 & 0 \\
0 & -1
\end{array}\right) .
\end{aligned}
$$

Первым интегрируемым многокомпонентным обобщением скалярного НУШ (1) стало векторное обобщение НУШ, предложенное Манаковым:

$$
\begin{aligned}
& i q_{1, t}+q_{1, x x}+2\left(\left|q_{1}\right|^{2}+\left|q_{2}\right|^{2}\right) q_{1}(x, t)=0, \\
& i q_{2, t}+q_{2, x x}+2\left(\left|q_{1}\right|^{2}+\left|q_{2}\right|^{2}\right) q_{2}(x, t)=0,
\end{aligned}
$$

где $q(x, t)$ - двухкомпонентная векторнозначная функция. Она ассоциируется с задачей рассеяния типа Захарова-Шабата, связанной с алгеброй $s l(3, \mathbb{C})$, и была предложена Манаковым [9] как асимптотическая модель распространения электрического поля в волноводе. Впоследствии эта система была получена как основная модель распространения света в оптических волокнах [10], [11].

Модель Манакова (3) обобщается на случай $n$-компонентных векторов (см. [3]):

$$
i \mathbf{v}_{t}+\mathbf{v}_{x x}+2\left(\mathbf{v}^{\dagger}, \mathbf{v}\right) \mathbf{v}=0, \quad \mathbf{v}=\mathbf{v}(x, t),
$$

где $\mathbf{v}-n$-компонентный комплекснозначный вектор, $(\cdot, \cdot)$ - стандартное скалярное произведение. Она также интегрируется с помощью МОЗР [2]-[5].

Классическая система Захарова-Шабата обобщается на случай матричной (многокомпонентной) формы разными способами. Один из стандартных методов такого обобщения - это рассмотрение операторов Лакса со значениями в простой алгебре Ли $\mathfrak{g}:$

$$
L=i \partial_{x}+Q-\lambda \mathrm{J}
$$

Здесь J - постоянный элемент простой алгебры Ли g [12], [13]. Обобщения НУШ на случай симметрических пространств, связанных с алгеброй Ли $\mathfrak{g}$, были предложены 
в работах [14]--[16]. К этим обобщениям относятся сопряженные НУШ системы с парами Лакса, связанные с симметрическими пространствами типа A.III, C.I, D.III и BD.I в классификации Картана [12], [17]. Этому классу также принадлежат как частные случаи векторное обобщение НУШ, предложенное Манаковым, и НУШ, изучавшееся в работе Кулиша и Склянина [18]. Все эти обобщения решаются с помощью МОЗР [4], [14], [18].

Когда ранг $r$ основополагающей простой алгебры Ли $\mathfrak{g}$ возрастает, число независимых комплекснозначных функций, содержащихся в соответствующих основных НЭУ (или системах НЭУ), равно полному числу корней алгебры g [19]-[21]. Они разрешимы при любых $r$, но их возможные физические приложения при больших $r$ не выглядят реалистическими. Однако все же можно получить новые интегрируемые и физически полезные НЭУ, проводя редукцию оператора $L(\lambda)$, т. е. налагая алгебраические условия на $Q(x, t)$, которые уменьшают количество независимых функций в них и количество уравнений [22]. Конечно, такие условия должны быть совместны с динамикой НЭУ [20], [21].

Недавно в работе [23] было предложено нелокальное интегрируемое уравнение типа НУШ

$$
i q_{t}+q_{x x}+V(x, t) q(x, t)=0, \quad V(x, t)=2 q(x, t) q^{*}(-x, t),
$$

обладающее $\mathcal{P} \mathcal{T}$-симметрией в силу инвариантности так называемого самоиндуцированного потенциала $V(x, t)$ относительно объединенного действия симметрий четности и обращения времени. В той же работе получено односолитонное решение для этой модели и показано, что в нем возникают особенности за конечное время. Вскоре после этого были найдены нелокальные $\mathcal{P} \mathcal{T}$-симметрические обобщения модели Абловица-Ладика в работе [24]. Все эти модели интегрируемы с помощью МОЗР [1], [25]-[27]. Такие нелокальные редукции НУШ и его многокомпонентные обобщения представляют значительный интерес для приложений в $\mathcal{P} \mathcal{T}$-симметричной оптике, особенно для развития теории электромагнитных волн в искусственных гетерогенных средах [28]-[31]. Обзор современных результатов можно найти, например, в работе [32].

Исторически первый псевдоэрмитов гамильтониан с действительным спектром это $\mathcal{P} \mathcal{T}$-симметрический гамильтониан из работы [33]. Здесь псевдоэрмитовость означает, что гамильтониан $\mathcal{H}$ коммутирует с операторами пространственного отражения $\mathcal{P}$ и обращения времени $\mathcal{T}$, т. е. $\mathcal{P} \mathcal{T} \mathcal{H}=\mathcal{H} \mathcal{P} \mathcal{T}$ [34], [35]. Эти операторы действуют следующим образом: $\mathcal{P}: x \rightarrow-x$ и $\mathcal{T}: t \rightarrow-t$ [36], [37]. Если волновая функция скалярна, то это приводит к следующему действию оператора пространственного отражения на пространстве состояний: $\mathcal{P} \psi(x, t)=\psi(-x, t)$ и $\mathcal{T} \psi(x, t)=$ $\psi^{*}(x,-t)$. В результате гамильтониан и волновая функция $\mathcal{P} \mathcal{T}$-симметричны, если $\mathcal{H}(x, t)=\mathcal{H}^{*}(-x,-t)$ и $\psi(x, t)=\psi^{*}(-x,-t)$ [38]. Здесь также учтено то обстоятельство, что оператор четности $\mathcal{P}$ линейный и унитарный, тогда как оператор обращения времени $\mathcal{T}$ антилинейный и антиунитарный.

Цель данной работы - изучить нелокальные редукции и получить соответствующие солитонные решения для многокомпонентных НУШ, которые связаны с симметрическими пространствами типов A.III и BD.I. Это будет сделано на основе примеров векторных НУШ, связанных с симметрическими пространствами типа 
A.III $\simeq S U(s+p) / S(U(s) \otimes U(p))$, и модели Кулиша-Склянина, связанной с симметрическими пространствами типа BD.I $\simeq S O(2 r+1) / S O(2) \otimes S O(2 r-1)$.

Статья организована следующим образом. В разделе 2 кратко представлены структура оператора Лакса, общий вид НЭУ и нелокальные симметрии (инволюции), имеющие определенный интерес. В разделе 3 рассматриваются прямая задача рассеяния, т. е. решения Йоста, матрица рассеяния и минимальное множество данных рассеяния, а также фундаментальные аналитические решения. В разделе 4 на основе подходящей модификации метода одевания Захарова-Шабата получены одно- и двухсолитонные решения соответствующего НЭУ, связанного с симметрическими пространствами типов A.III и BD.I, и изучен эффект нелокальной редукции на них.

\section{2. ПРЕДВАРИТЕЛЬНЫЕ СВЕДЕНИЯ}

2.1. Пара Лакса и общий вид уравнений. Начнем с основной пары Лакса для многокомпонентных НУШ в симметрических пространствах [39], ее можно представить в следующем виде:

$$
\begin{aligned}
L \chi(x, t, \lambda) & \equiv i \partial_{x} \chi+(Q(x, t)-\lambda \mathrm{J}) \chi(x, t, \lambda)=0, \quad U(x, t, \lambda)=Q(x, t)-\lambda \mathrm{J}, \\
M \chi(x, t, \lambda) & \equiv i \partial_{t} \chi+\left(V_{0}(x, t)+\lambda V_{1}(x, t)-\lambda^{2} \mathrm{~J}\right) \chi(x, t, \lambda)=0, \\
V_{1}(x, t) & =Q(x, t), \quad V_{0}(x, t)=i \operatorname{ad}_{\mathrm{J}}^{-1} \frac{d Q}{d x}+\frac{1}{2}\left[\operatorname{ad}_{\mathrm{J}}^{-1} Q, Q(x, t)\right] .
\end{aligned}
$$

Здесь $\chi(x, t, \lambda)$ - собственная функция операторов Лакса, $U(x, t, \lambda)$ и $V(x, t, \lambda)$ выбраны так, чтобы они принимали значения в простой алгебре Ли g ранга $r$, тогда как собственные функции $\chi(x, t, \lambda)$ принадлежат соответствующей группе Ли $\mathfrak{G}$. Здесь также J - постоянный элемент подалгебры Картана $\mathfrak{h} \subset \mathfrak{g}$ (который всегда можно выбрать так, чтобы он представлялся диагональной матрицей), $\lambda \in \mathbb{C}$ - спектральный параметр. На симметрическом пространстве типа $\mathbf{A} . \mathbf{I I I} \simeq$ $S U(p+s) / S(U(p) \times U(s))$ потенциал оператора $L(\lambda)(7$ а) имеет вид

$$
Q(x, t)=\left(\begin{array}{cc}
0 & \mathbf{q}^{+} \\
\mathbf{q}^{-} & 0
\end{array}\right), \quad \mathrm{J}=\frac{2}{s+p}\left(\begin{array}{cc}
p \mathbb{1}_{s} & 0 \\
0 & -s \mathbb{1}_{p}
\end{array}\right),
$$

где $\mathbf{q}^{+}(x, t)$ и $\left(\mathbf{q}^{-}\right)^{\mathrm{T}}(x, t)-(s \times p)$-матричнозначные функции, принадлежащие простой алгебре Ли $\mathfrak{g}, \mathbb{1}_{s}$ и $\mathbb{1}_{p}-$ соответственно $(s \times s)$ - и $(p \times p)$-единичные матрицы, $s+p=n$.

НЭУ можно представить как условие совместности

$$
[L(\lambda), M(\lambda)]=0
$$

двух операторов Лакса (7a), (7б). В частности, если $L(\lambda)$ и $M(\lambda)$ связаны с симметрическими пространствами типа $\mathbf{A . I I I} \simeq S U(p+1) / S(U(1) \otimes U(p))$ [14], то явная параметризация (8) симметрических пространств типа A.III приводит к системе

$$
\begin{aligned}
i \mathbf{q}_{t}^{+}+\mathbf{q}_{x x}^{+}+2 \mathbf{q}^{+} \mathbf{q}^{-} \mathbf{q}^{+}(x, t) & =0 \\
-i \mathbf{q}_{t}^{-}+\mathbf{q}_{x x}^{-}+2 \mathbf{q}^{-} \mathbf{q}^{+} \mathbf{q}^{-}(x, t) & =0 .
\end{aligned}
$$


Частный случай $s=1$ и $p=2$ (также предполагающий стандартную инволюцию $\mathbf{q}^{-}=\left(\mathbf{q}^{+}\right)^{*}$ для моделей типа НУШ) соответствует хорошо известной системе Манакова [9]. Ее обобщение на случай $n$-мерных векторов $\mathbf{q}^{ \pm}$известно как векторное НУШ [4].

Другой класс многокомпонентных НУШ известен как модели Кулиша-Склянина, которые связаны с симметрическими пространствами типа BD.I $\simeq S O(2 r+1) / S O(2) \otimes$ $S O(2 r-1)[40]-[44]$. Основные НЭУ этого класса можно представить в виде

$$
\begin{aligned}
& i \mathbf{q}_{t}^{+}+\mathbf{q}_{x x}^{+}+2\left(\mathbf{q}^{+}, \mathbf{q}^{-}\right) \mathbf{q}^{+}-\left(\mathbf{q}^{+}, s_{0} \mathbf{q}^{+}\right) s_{0} \mathbf{q}^{-}=0, \\
& i \mathbf{q}_{t}^{-}-\mathbf{q}_{x x}^{-}-2\left(\mathbf{q}^{+}, \mathbf{q}^{-}\right) \mathbf{q}^{-}+\left(\mathbf{q}^{-}, s_{0} \mathbf{q}^{-}\right) s_{0} \mathbf{q}^{+}=0,
\end{aligned}
$$

который ассоциируется с линейной системой $(7 \mathrm{a})$ алгебры $\mathfrak{g} \simeq s o(2 r+1, \mathbb{C})$, где

$$
Q(x, t)=\left(\begin{array}{ccc}
0 & \left(\mathbf{q}^{+}\right)^{\mathrm{T}} & 0 \\
\mathbf{q}^{-} & \mathbf{0} & s_{0} \mathbf{q}^{+} \\
0 & \left(\mathbf{q}^{-}\right)^{\mathrm{T}} s_{0} & 0
\end{array}\right), \quad \mathrm{J}=\operatorname{diag}(1, \mathbf{0},-1) .
$$

Здесь $\mathbf{q}^{ \pm}-(2 r-1)$-компонентные векторы, $s_{0}$ - матрица, определяющая ортогональную алгебру $\mathbf{B}_{r} \simeq s o(2 r+1)$ :

$$
\begin{aligned}
& X \in s o(2 r+1) \quad \text { тогда и только тогда, когда } X+S_{0} X^{\mathrm{T}} S_{0}^{-1}=0, \\
& S_{0}=\sum_{s=1}^{2 r+1}(-1)^{s+1} E_{s, 2 r+2-s}^{(2 r+1)}=\left(\begin{array}{ccc}
0 & 0 & 1 \\
0 & -s_{0} & 0 \\
1 & 0 & 0
\end{array}\right),
\end{aligned}
$$

где $E_{s, p}^{(2 r+1)}$ - матрица размера $(2 r+1 \times 2 r+1)$, элементы которой заданы выражениями $\left(E_{s, p}^{(2 r+1)}\right)_{i j}=\delta_{s i} \delta_{p j}$.

2.2. Симметрии оператора Лакса и редукции. Систематический метод описания и классификации класса допустимых редукций для заданной пары Лакса был предложен Михайловым [22]. Этот метод основан на применении так называемой группы редукций. Группа редукций - это конечная группа, сохраняющая представление Лакса, т. е. она обеспечивает автоматическую совместность ограничений редукции с эволюцией [20], [21], [45], [46]. Условия редукции уменьшают число независимых функций и число уравнений. Здесь мы ограничимся рассмотрением $\mathbb{Z}_{2}$-редукций двух типов:

$$
\begin{aligned}
(\mathrm{A}): & U(x, t, \lambda) & =B\left(U\left(x, t, \lambda^{*}\right)\right)^{\dagger} B^{-1}, \\
(\mathrm{~B}): & -U(x, t, \lambda) & =B\left(U\left(-x, t,-\lambda^{*}\right)\right)^{\dagger} B^{-1},
\end{aligned}
$$

где $B$ - постоянная блочно-диагональная матрица вида $B=\left(\begin{array}{cc}\mathbf{B}_{+} & 0 \\ 0 & \mathbf{B}_{-}\end{array}\right)$для симметрических пространств типа A.III. Для симметрических пространств типа BD.I эту матрицу можно представить в виде

$$
B=\left(\begin{array}{ccc}
\mathbf{B}_{+} & 0 & 0 \\
0 & \mathbf{B}_{ \pm} & 0 \\
0 & 0 & \mathbf{B}_{-}
\end{array}\right)
$$


Здесь также предполагается, что блоки $\mathbf{B}_{+}$и $\mathbf{B}_{-}-$несингулярные матрицы. Также заметим, что инволюция типа (В) в (16) приводит к нелокальным условиям редукции.

В силу стандартной блочной структуры в симметрических пространствах типа A.III (8) условия редукции для матричных блоков можно представить в явном виде:

$$
\begin{array}{ll}
(\mathrm{A}): \mathbf{q}^{-}(x, t)=\mathbf{B}_{-}\left(\mathbf{q}^{+}(x, t)\right)^{\dagger}\left(\mathbf{B}_{+}\right)^{-1}, & \mathbf{q}^{+}(x, t)=\mathbf{B}_{+}\left(\mathbf{q}^{-}(x, t)\right)^{\dagger}\left(\mathbf{B}_{-}\right)^{-1} \\
(\mathrm{~B}): \mathbf{q}^{-}(x, t)=-\mathbf{B}_{-}\left(\mathbf{q}^{+}(-x, t)\right)^{\dagger}\left(\mathbf{B}_{+}\right)^{-1}, & \mathbf{q}^{+}(x, t)=-\mathbf{B}_{+}\left(\mathbf{q}^{-}(-x, t)\right)^{\dagger}\left(\mathbf{B}_{-}\right)^{-1} .
\end{array}
$$

Применяя соответственно локальные и нелокальные инволюции (18) и (19), можно записать полученные модели Кулиша-Склянина. Если взять В как единичную матрицу, то локальная инволюция (18) дает оценки

$$
i \mathbf{q}_{t}^{+}+\mathbf{q}_{x x}^{+}+2\left(\mathbf{q}^{+}(x, t),\left(\mathbf{q}^{+}(x, t)\right)^{*}\right) \mathbf{q}^{+}-\left(\mathbf{q}^{+}, s_{0} \mathbf{q}^{+}\right) s_{0}\left(\mathbf{q}^{+}(x, t)\right)^{*}=0,
$$

тогда как нелокальная инволюция (19) дает

$$
i \mathbf{q}_{t}^{+}+\mathbf{q}_{x x}^{+}+2\left(\mathbf{q}^{+}(x, t),\left(\mathbf{q}^{+}(-x, t)\right)^{*}\right) \mathbf{q}^{+}-\left(\mathbf{q}^{+}, s_{0} \mathbf{q}^{+}\right) s_{0}\left(\mathbf{q}^{+}(-x, t)\right)^{*}=0 .
$$

ПрИМЕР 1 (МОДЕЛЬ МАНАКОВА). Если $\mathfrak{g} \simeq \operatorname{sl}(3, \mathbb{C}), p=1$ и $s=2$, то $\mathbf{q}^{+}(x, t)$ и $\mathbf{q}^{-}(x, t)$ - двухкомпонентные векторные функции. Кроме того, если применить (локальную) редукцию типа (А) при $B=\mathbb{I}$, то получится стандартное векторное обобщение НУШ, предложенное Манаковым:

$$
\begin{aligned}
& -i q_{1, t}+q_{1, x x}+2\left(\left|q_{1}(x, t)\right|^{2}+\left|q_{2}(x, t)\right|^{2}\right) q_{1}(x, t)=0, \\
& -i q_{2, t}+q_{2, x x}+2\left(\left|q_{1}(x, t)\right|^{2}+\left|q_{2}(x, t)\right|^{2}\right) q_{2}(x, t)=0 .
\end{aligned}
$$

Применяя инволюцию типа (В) при $\mathbf{B}_{+}=\mp 1$ и $\mathbf{B}_{-}=\operatorname{diag}( \pm 1, \pm 1)$, получим следующую нелокальную редукцию модели Манакова:

$$
\begin{aligned}
& -i q_{1, t}+q_{1, x x}+2\left(q_{1}(x, t) q_{1}^{*}(-x, t)+q_{2}(x, t) q_{2}^{*}(-x, t)\right) q_{1}(x, t)=0 \\
& -i q_{2, t}+q_{2, x x}+2\left(q_{1}(x, t) q_{1}^{*}(-x, t)+q_{2}(x, t) q_{2}^{*}(-x, t)\right) q_{2}(x, t)=0 .
\end{aligned}
$$

ПримеР 2 (мОДЕЛЬ КУЛИшА-СКЛЯНинА). В простейшем случае операторов Лакса, связанных с $S O(5) / S O(2) \otimes S O(3)(\operatorname{rank} \mathfrak{g}=2)$, можно положить $\mathbf{q}^{+}=$ $\left(q_{12}^{+}, q_{13}^{+}, q_{14}^{+}\right)$и $\mathbf{q}^{-}=\left(q_{12}^{-}, q_{13}^{-}, q_{14}^{-}\right)$. Также, применяя стандартную инволюцию типа (A) при $B=\mathbb{I}$, получим, что условие совместности (9) приводит к следующей трехкомпонентной системе НУШ:

$$
\begin{array}{r}
i\left(q_{t}^{+}\right)_{12}+\left(q_{x x}^{+}\right)_{12}+2\left(\left|q_{12}\right|^{2}+2\left|q_{13}\right|^{2}\right) q_{12}+2\left(q_{14}^{+}\right)^{*}\left(q_{13}^{+}\right)^{2}=0 \\
i\left(q_{t}^{+}\right)_{13}+\left(q_{x x}^{+}\right)_{13}+2\left(\left|q_{12}\right|^{2}+\left|q_{13}\right|^{2}+\left|q_{14}\right|^{2}\right) q_{13}+2\left(q_{13}^{+}\right)^{*} q_{14}^{+} q_{12}^{+}=0 \\
i\left(q_{t}^{+}\right)_{14}+\left(q_{x x}^{+}\right)_{14}+2\left(\left|q_{14}\right|^{2}+2\left|q_{13}\right|^{2}\right) q_{14}+2\left(q_{12}^{+}\right)^{*}\left(q_{13}^{+}\right)^{2}=0 .
\end{array}
$$

Это оказывается моделью, описывающей $\mathcal{F}=1$ спинорные конденсаты Бозе-Эйнштейна в одномерной аппроксимации [19], [44], [47], [48]. Аналогичная система была представлена в [49], при этом использовалась эквивалентная редукция. 


\section{3. ПРЯМОЕ ПРЕОБРАЗОВАНИЕ РАССЕЯНИЯ ПРИ $L(\lambda)$}

3.1. Решения Йоста и матрица рассеяния. Начнем с так называемых решений Йоста, которые определяются своими асимптотиками при $|x| \rightarrow \infty$ :

$$
\lim _{x \rightarrow \infty} e^{i \lambda \mathrm{J} x} \psi(x, t, \lambda)=\mathbb{1}_{s+p}, \quad \lim _{x \rightarrow-\infty} e^{i \lambda \mathrm{J} x} \phi(x, t, \lambda)=\mathbb{1}_{s+p}, \quad \lambda \in \mathbb{R} .
$$

Вместе с этими функциями также применяются решения Йоста, нормированные на единицу,

$$
\xi(x, t, \lambda)=\psi(x, t, \lambda) e^{i \lambda \mathrm{J} x}, \quad \varphi(x, t, \lambda)=\phi(x, t, \lambda) e^{i \lambda \mathrm{J} x},
$$

которые удовлетворяют уравнению

$$
i \frac{d \xi}{d x}+Q(x, t) \xi(x, t, \lambda)-\lambda[\mathrm{J}, \xi(x, t, \lambda)]=0,
$$

при условии, что асимптотики (25) удовлетворяют (7а).

На непрерывном спектре оператора $L(\lambda)$ два решения Йоста $\psi(x, t, \lambda)$ и $\phi(x, t, \lambda)$ связаны матрицей рассеяния $T(\lambda)$ :

$$
\phi(x, t, \lambda)=\psi(x, t, \lambda) T(\lambda), \quad \lambda \in \mathbb{R} .
$$

Матрица рассеяния $T(\lambda)$ принадлежит группе Ли $\mathfrak{G}$, соответствующей алгебре Ли, или симметрическому пространству оператора $L(\lambda)$. В случае симметрических пространств типа A.III в классификации Картана она имеет блочную структуру:

$$
T(\lambda)=\left(\begin{array}{cc}
\mathbf{a}^{+}(\lambda) & -\mathbf{b}^{-}(\lambda) \\
\mathbf{b}^{+}(\lambda) & \mathbf{a}^{-}(\lambda)
\end{array}\right)
$$

где $\mathbf{a}^{+}(\lambda)$ и $\mathbf{a}^{-}(\lambda)$ - квадратные матрицы, тогда как $\mathbf{b}^{+}(\lambda)$ и $\mathbf{b}^{-}(\lambda)$ - прямоугольные матрицы. Блочная структура матрицы, обратной к $T(\lambda)$, имеет вид

$$
\widehat{T}(\lambda)=\left(\begin{array}{cc}
\mathbf{c}^{-}(\lambda) & \mathbf{d}^{-}(\lambda) \\
-\mathbf{d}^{+}(\lambda) & \mathbf{c}^{+}(\lambda)
\end{array}\right), \quad \widehat{T}(\lambda)=T^{-1}(\lambda),
$$

где

$$
\begin{aligned}
\mathbf{c}^{ \pm}(\lambda) & =\hat{\mathbf{a}}^{\mp}(\lambda)\left(\mathbb{1}+\rho^{ \pm} \rho^{\mp}\right)^{-1}=\left(\mathbb{1}+\tau^{\mp} \tau^{ \pm}\right)^{-1} \hat{\mathbf{a}}^{\mp}(\lambda), \\
\mathbf{d}^{ \pm}(\lambda) & =\hat{\mathbf{a}}^{\mp}(\lambda) \rho^{ \pm}(\lambda)\left(\mathbb{1}+\rho^{\mp} \rho^{ \pm}\right)^{-1}=\left(\mathbb{1}+\tau^{\mp} \tau^{ \pm}\right)^{-1} \tau^{\mp}(\lambda) \hat{\mathbf{a}}^{ \pm}(\lambda) .
\end{aligned}
$$

Здесь $\rho^{ \pm}(\lambda)$ и $\tau^{ \pm}(\lambda)$ - коэффициенты отражения и пропускания соответственно:

$$
\rho^{ \pm}(\lambda)=\mathbf{b}^{ \pm} \hat{\mathbf{a}}^{ \pm}(\lambda)=\hat{\mathbf{c}}^{ \pm} \mathbf{d}^{ \pm}(\lambda), \quad \tau^{ \pm}(\lambda)=\hat{\mathbf{a}}^{ \pm} \mathbf{b}^{\mp}(\lambda)=\mathbf{d}^{\mp} \hat{\mathbf{c}}^{ \pm}(\lambda) .
$$

В случае симметрических пространств типа BD.I блочная структура матрицы $T(t, \lambda)$ и матрицы, обратной к ней, имеет вид

$$
T(t, \lambda)=\left(\begin{array}{ccc}
m_{1}^{+} & -\overrightarrow{\mathbf{B}}^{-T} & c_{(1)}^{-} \\
\overrightarrow{\mathbf{b}}^{+} & \mathbf{T}_{22} & -s_{0} \overrightarrow{\mathbf{b}}^{-} \\
c_{(1)}^{+} & \overrightarrow{\mathbf{B}}^{+T} s_{0} & m_{1}^{-}
\end{array}\right), \quad \widehat{T}(t, \lambda)=\left(\begin{array}{ccc}
m_{1}^{-} & \overrightarrow{\mathbf{b}}^{-T} & c_{(1)}^{-} \\
-\overrightarrow{\mathbf{B}}^{+} & \widehat{\mathbf{T}}_{22} & s_{0} \overrightarrow{\mathbf{B}}^{-} \\
c_{(1)}^{+} & -\overrightarrow{\mathbf{b}}^{+T} s_{0} & m_{1}^{+}
\end{array}\right),
$$

где $\overrightarrow{\mathbf{B}}^{ \pm}(t, \lambda)$ и $\overrightarrow{\mathbf{b}}^{ \pm}(t, \lambda)-(2 r-1)$-компонентные векторы, а $c_{(1)}^{ \pm}(\lambda)$ и $m_{1}^{ \pm}(\lambda)$ - скалярные функции. Коэффициенты отражения $\vec{\rho}^{ \pm}(\lambda)$, коэффициенты пропускания $\vec{\tau}^{ \pm}(\lambda)$ и функции $c_{(1)}^{ \pm}(\lambda)$ определяются обобщенным разложением Гаусса матрицы

$$
T(t, \lambda)=T_{\mathrm{J}}^{-}(t, \lambda) D_{\mathrm{J}}^{+}(t, \lambda) \widehat{S}_{\mathrm{J}}^{+}(t, \lambda)=T_{\mathrm{J}}^{+}(t, \lambda) D_{\mathrm{J}}^{-}(t, \lambda) \widehat{S}_{\mathrm{J}}^{-}(t, \lambda) .
$$


Здесь $S_{\mathrm{J}}^{ \pm}$и $T_{\mathrm{J}}^{ \pm}$- блочные верхне- и нижнетреугольная матрицы, которые можно представить в виде

$$
S_{\mathrm{J}}^{ \pm}=e^{\left( \pm\left(\vec{\tau}^{ \pm}(\lambda, t) \cdot \vec{E}_{1}^{ \pm}\right)\right)}, \quad T_{\mathrm{J}}^{ \pm}=e^{\left(\mp\left(\vec{\rho}^{\mp}(\lambda, t) \cdot \vec{E}_{1}^{ \pm}\right)\right)},
$$

где

$$
\vec{\tau}^{ \pm}(\lambda, t)=\frac{\overrightarrow{\mathbf{B}}^{\mp}}{m_{1}^{ \pm}}, \quad \vec{\rho}^{ \pm}(\lambda, t)=\frac{\overrightarrow{\mathbf{b}}^{ \pm}}{m_{1}^{ \pm}}, \quad c_{(1)}^{ \pm}=\frac{m_{1}^{ \pm}}{2}\left(\vec{\rho}^{ \pm, \mathrm{T}}, s_{0} \vec{\rho}^{ \pm}\right)=\frac{m_{1}^{\mp}}{2}\left(\vec{\tau}^{\mp, \mathrm{T}}, s_{0} \vec{\tau}^{\mp}\right),
$$

$D_{\mathrm{J}}^{ \pm}(t, \lambda)$ - блочно-диагональный множитель в $(34)$ :

$$
D_{\mathrm{J}}^{+}=\left(\begin{array}{ccc}
m_{1}^{+} & 0 & 0 \\
0 & \mathbf{m}_{2}^{+} & 0 \\
0 & 0 & 1 / m_{1}^{+}
\end{array}\right), \quad D_{\mathrm{J}}^{-}=\left(\begin{array}{ccc}
1 / m_{1}^{-} & 0 & 0 \\
0 & \mathbf{m}_{2}^{-} & 0 \\
0 & 0 & m_{1}^{-}
\end{array}\right)
$$

где $m_{k}^{ \pm}(t, \lambda)$ - верхний и нижний главные миноры ранга $k$ матрицы $T(t, \lambda)(34)$ и

$$
\mathbf{m}_{2}^{+}=\mathbf{T}_{22}+\frac{\vec{b}^{+} \vec{B}^{-, T}}{m_{1}^{+}}, \quad \mathbf{m}_{2}^{-}=\mathbf{T}_{22}+\frac{s_{0} \vec{B}^{-} \vec{b}^{+, \mathrm{T}} s_{0}}{m_{1}^{-}}
$$

Если матрица потенциала $Q(x, t)$ удовлетворяет НЭУ $(9)$, то ассоциированная матрица рассеяния $T(t, \lambda)$ меняется линейно по времени, т. е. удовлетворяет уравнению

$$
i \frac{d T}{d t}+[f(\lambda), T(t, \lambda)]=0,
$$

где $f(\lambda)$ - закон дисперсии для НЭУ (9). Для уравнений типа НУШ $f(\lambda)=-\lambda^{2} J$.

3.2. Фундаментальные аналитические решения. Обрисуем коротко построение фундаментальных аналитических решений $\chi(x, t, \lambda)$ обобщенной системы Захарова-Шабата (7) [39], [4]. Их можно получить непосредственно из решений Йоста для системы (7):

$$
\chi^{ \pm}(x, t, \lambda)=\phi(x, t, \lambda) S_{\mathrm{J}}^{ \pm}(t, \lambda)=\psi(x, t, \lambda) T_{\mathrm{J}}^{\mp}(t, \lambda) D_{\mathrm{J}}^{ \pm}(t, \lambda),
$$

с помощью обобщенного разложения Гаусса (34) матрицы рассеяния $T(t, \lambda)$.

На действительной оси (т. е. на непрерывном спектре оператора $L(\lambda))$ два фундаментальных аналитических решения линейно зависимы:

$$
\chi^{+}(x, t, \lambda)=\chi^{-}(x, t, \lambda) G_{0}(t, \lambda), \quad \lambda \in \mathbb{R},
$$

где сшивающая функция $G_{0}(t, \lambda)$ выражается через обобщенные множители Гаусса $S_{\mathrm{J}}^{ \pm}(t, \lambda)$ следующим образом:

$$
G_{0, J}(t, \lambda)=\left.\widehat{S}_{J}^{-}(t, \lambda) S_{J}^{+}(t, \lambda)\right|_{t=0} .
$$

Независимые элементы матрицы $G_{0}(t, \lambda)$ вместе с дискретным спектром оператора $L(\lambda)$ образуют минимальное множество данных рассеяния оператора $L$. На основе соотношений полноты для ассоциированных “квадратов решений” и соотношений 
типа Вронского можно восстановить потенциал $Q(x, t)$ по минимальному множеству данных рассеяния [49].

В завершение этого раздела заметим, что хотя общий вид разложения Гаусса (34) имеет место и в симметрическом пространстве, для симметрических пространств типа A.III можно упростить вид матричного блока, слегка изменив разложение Гаусса матрицы $T(\lambda)^{1)}$ :

$$
T(\lambda)=\mathbf{T}_{J}^{-}(\lambda) \widehat{\mathbf{S}}_{J}^{+}(\lambda)=\mathbf{T}_{J}^{+}(\lambda) \widehat{\mathbf{S}}_{J}^{-}(\lambda) .
$$

Теперь $\mathbf{S}^{ \pm}(\lambda)$ и $\mathbf{T}^{ \pm}(\lambda)$ - блочно-треугольные матрицы:

$$
\begin{array}{ll}
\mathbf{S}_{J}^{+}(\lambda)=\left(\begin{array}{cc}
\mathbb{1}_{s}, & \mathbf{d}^{-}(\lambda) \\
0, & \mathbf{c}^{+}(\lambda)
\end{array}\right), & \mathbf{T}_{J}^{-}(\lambda)=\left(\begin{array}{cc}
\mathbf{a}^{+}(\lambda), & 0 \\
\mathbf{b}^{+}(\lambda), & \mathbb{1}_{p}
\end{array}\right), \\
\mathbf{S}_{J}^{-}(\lambda)=\left(\begin{array}{cc}
\mathbf{c}^{-}(\lambda), & 0 \\
-\mathbf{d}^{+}(\lambda), & \mathbb{1}_{p}
\end{array}\right), & \mathbf{T}_{J}^{+}(\lambda)=\left(\begin{array}{cc}
\mathbb{1}_{s}, & -\mathbf{b}^{-}(\lambda) \\
0, & \mathbf{a}^{-}(\lambda)
\end{array}\right) .
\end{array}
$$

Применяя разложение Гаусса (43), выражаем сшивающую функцию $G_{0, J}(t, \lambda)(42)$ через блоки матрицы $T(t, \lambda)$ и матрицы, обратной к ней, в явном виде:

$$
G_{0}(\lambda)=\widehat{D}^{-}(\lambda)\left(\mathbb{1}+K^{-}(\lambda)\right), \quad \widehat{G}_{0}(\lambda)=\widehat{D}^{+}(\lambda)\left(\mathbb{1}-K^{+}(\lambda)\right) .
$$

Здесь блочно-диагональные множители $D^{ \pm}(\lambda)$ и блочно-внедиагональные множители $K^{ \pm}(\lambda)$ выражаются через блоки $\mathbf{a}^{ \pm}(\lambda)$ и $\mathbf{b}^{ \pm}(\lambda)$ матрицы $T(\lambda)(29)$ и через блоки $\mathbf{c}^{ \pm}(\lambda)$ и $\mathbf{d}^{ \pm}(\lambda)(30)$ обратной матрицы $\widehat{T}(\lambda)$ соответственно:

$$
\begin{aligned}
& D^{-}(\lambda)=\left(\begin{array}{cc}
\mathbf{c}^{-}(\lambda) & 0 \\
0 & \mathbf{a}^{-}(\lambda)
\end{array}\right), \quad K^{-}(\lambda)=\left(\begin{array}{cc}
0 & \mathbf{d}^{-}(\lambda) \\
\mathbf{b}^{+}(\lambda) & 0
\end{array}\right), \\
& D^{+}(\lambda)=\left(\begin{array}{cc}
\mathbf{a}^{+}(\lambda) & 0 \\
0 & \mathbf{c}^{+}(\lambda)
\end{array}\right), \quad K^{+}(\lambda)=\left(\begin{array}{cc}
0 & \mathbf{b}^{-}(\lambda) \\
\mathbf{d}^{+}(\lambda) & 0
\end{array}\right) \text {. }
\end{aligned}
$$

Верхние индексы \pm в представленных выше выражениях $D^{ \pm}(\lambda)$ означают аналитичность по $\lambda \in \mathbb{C}_{ \pm}$.

\section{4. МЕТОД ОДЕВАНИЯ И СОЛИТОННЫЕ РЕШЕНИЯ}

4.1. Метод одевания Захарова-Шабата. Основная идея метода одевания Захарова-Шабата заключается в том, чтобы начать с регулярного решения $\eta_{0}^{ \pm}(x, t, \lambda)$ уравнения (7a) и осуществить одевание, добавляя сингулярности в заданных точ$\operatorname{каx} \lambda_{k}^{ \pm}[3],[39]$, [40], [50]-[54]. Новое сингулярное решение будет иметь вид

$$
\eta^{ \pm}(x, t, \lambda)=u_{k}(x, t, \lambda) \eta_{0}^{ \pm}(x, t, \lambda) w_{k, \pm}^{-1}(\lambda)
$$

Здесь матрицы $w_{k, \pm}(\lambda)$ задаются выражениями

$$
w_{k,+}(\lambda)=\mathbb{1}, \quad w_{k,-}(\lambda)=\left(\begin{array}{cc}
u_{11, k}^{+} & 0 \\
0 & u_{22, k}^{-}
\end{array}\right),
$$

1) Разложение типа (43) известно как LU-разложение матрицы $T(\lambda)$, тогда как разложение типа (34) известно как LDU-разложение матрицы $T(\lambda)$. 
где пределы одевающего множителя следующие:

$$
\begin{aligned}
\lim _{x \rightarrow \infty} u_{k}(x, t, \lambda)=\left(\begin{array}{cc}
u_{11, k}^{+} & 0 \\
0 & \mathbb{1}
\end{array}\right), & u_{11, k}^{+}=\mathbb{1}+\left(c_{k}(\lambda)-1\right) P_{11, k}^{+}, \\
\lim _{x \rightarrow-\infty} u_{k}(x, t, \lambda)=\left(\begin{array}{cc}
\mathbb{1} & 0 \\
0 & u_{22, k}^{-}
\end{array}\right), & u_{22, k}^{-}=\mathbb{1}+\left(c_{k}(\lambda)-1\right) P_{22, k}^{-} .
\end{aligned}
$$

Для операторов Лакса, связанных с симметрическими пространствами типа A.III, одевающие множители имеют вид

$$
u_{k}(x, t, \lambda)=\mathbb{1}+\left(c_{k}(\lambda)-1\right) P_{k}(x, t), \quad c_{k}(\lambda)=\frac{\lambda-\lambda_{k}^{+}}{\lambda-\lambda_{k}^{-}} .
$$

Здесь $u_{k}(x, t, \lambda)$ - одевающий множитель, $P_{k}(x, t)$ - проектор ранга 1 :

$$
P_{k}(x, t)=\frac{\left|n_{k}(x, t)\right\rangle\left\langle m_{k}(x, t)\right|}{\left\langle m_{k}(x, t) \mid n_{k}(x, t)\right\rangle},
$$

где $\left|n_{k}(x, t)\right\rangle=\chi_{0}^{+}\left(x, t, \lambda_{1}^{+}\right)\left|n_{0,1}\right\rangle$ - вектор-столбец, $\left\langle m_{1}(x, t)\right|=\left\langle m_{0,1}\right| \widehat{\chi}_{0}^{-}\left(x, t, \lambda_{1}^{-}\right)-$ вектор-строка, и они оба являются собственными векторами. Проекторы $P_{k}(x, t)$ автоматически удовлетворяют условию $P_{k}^{2}(x, t)=P_{k}(x, t)$. Функции $\eta^{ \pm}(x, t, \lambda)$ удовлетворяют линейной системе

$$
\left(i \frac{d \eta^{ \pm}}{d x}+Q(x, t) \eta^{ \pm}(x, t, \lambda)-\lambda\left[\mathrm{J}, \eta^{ \pm}(x, t, \lambda)\right]\right)=0
$$

и связаны с системой Захарова-Шабата с неизвестным потенциалом $Q(x, t)$, который мы определим ниже, тогда как $\eta_{0}^{ \pm}(x, t, \lambda)$ связаны с системой Захарова-Шабата с известным потенциалом $Q_{0}(x, t)$ :

$$
i \frac{d \eta_{0}^{ \pm}}{d x}+Q_{0}(x, t) \eta_{0}^{ \pm}(x, t, \lambda)-\lambda\left[\mathrm{J}, \eta_{0}^{ \pm}(x, t, \lambda)\right]=0 .
$$

Теперь из (47) и (52) находим, что одевающий множитель $u_{k}(x, t, \lambda)$ удовлетворяет уравнению

$$
i \frac{d u_{k}}{d x}+Q(x, t) u_{k}(x, t, \lambda)-u_{k}(x, t, \lambda) Q_{0}(x, t)-\lambda\left[\mathrm{J}, u_{k}(x, t, \lambda)\right]=0 .
$$

Поскольку анзацы для одевающего множителя $u_{k}(x, t, \lambda)$ в $(49)$ и $(53)$ совместны по $\lambda$, существуют два условия, которые можно применить к левой части уравнения (53) и которые дают предел при $\lambda \rightarrow \infty$ и вычет в точке $\lambda=\lambda_{k}^{-}$, и они оба обращаются в нуль. Первое условие приводит к потенциалу

$$
Q(x, t)-Q_{0}(x, t)=-\left(\lambda_{k}^{+}-\lambda_{k}^{-}\right)\left[\mathrm{J}, P_{k}(x, t)\right],
$$

а второе условие дает нелинейное уравнение для $P_{k}(x, t)$ :

$$
\begin{aligned}
i \frac{d P_{k}}{d x} & +Q_{0}(x, t) P_{k}(x, t)-P_{k}(x, t) Q_{0}(x, t)-\lambda_{k}^{+} \mathrm{J} P_{k}(x, t)+\lambda_{k}^{-} P_{k}(x, t) \mathrm{J}+ \\
& +\left(\lambda_{k}^{+}-\lambda_{k}^{-}\right) P_{k}(x, t) \mathrm{J} P_{k}(x)=0 .
\end{aligned}
$$

Кроме того, имеется условие нормировки $\lim _{\lambda \rightarrow \infty} u_{k}(x, t, \lambda)=\mathbb{1}$. 
В случае симметрических пространств типа BD.I одевающий множитель $u_{k}(x, t, \lambda)$ можно представить в виде

$$
u_{k}(x, t, \lambda)=\mathbb{1}+\left(c_{k}(\lambda)-1\right) P_{k}(x, t)+\left(\frac{1}{c_{k}(\lambda)}-1\right) \bar{P}_{k}(x, t), \quad \bar{P}_{k}=S_{0} P_{k}^{\mathrm{T}} S_{0}^{-1},
$$

где $P_{k}(x, t)$ и $\bar{P}_{k}(x, t)$ - взаимно ортогональные проекторы ранга 1 [21]. Аналогично можно представить одетый потенциал $Q(x, t)$ как

$$
Q(x, t)=Q_{0}(x, t)-\left(\lambda_{k}^{+}-\lambda_{k}^{-}\right)\left[\mathrm{J}, P_{k}(x, t)-\bar{P}_{k}(x, t)\right] .
$$

\section{2. Односолитонные решения.}

Пример 3. Если взять $\mathfrak{g} \simeq \operatorname{sl}(3, \mathbb{C})$ и одевающий множитель в виде $(49)$, Удовлетворяющем нелокальным условиям редукции (16), то получим следующую инволюцию одевающего множителя:

$$
B u_{1}\left(-x, t,-\lambda^{*}\right)^{\dagger} B^{-1}=u_{1}^{-1}(x, t, \lambda),
$$

где $B$ - постоянная блочно-диагональная матрица. В результате проектор $P_{1}$ должен удовлетворять условию

$$
P_{1}(x, t)=B P_{1}^{\dagger}(-x, t) B^{-1}, \quad\left(-\lambda^{ \pm}\right)^{*}=\lambda^{\mp} .
$$

Это значит, что проектор $P_{1}(x, t)$ и $c_{1}(\lambda)$ принимают вид

$$
\begin{gathered}
P_{1}(x, t)=\frac{\left|n_{1}(x, t)\right\rangle\left\langle n_{1}^{*}(-x, t)\right| B}{\left\langle n_{1}^{*}(-x, t)|B| n_{1}(x, t)\right\rangle}, \quad\left\langle m_{1}(x, t)\right|=\left(B\left|n_{1}(-x, t)\right\rangle\right)^{\dagger}, \\
c_{1}(\lambda)=\frac{\lambda-\lambda_{1}^{+}}{\lambda+\left(\lambda_{1}^{+}\right)^{*}} .
\end{gathered}
$$

Итак, из (54) следует, что компоненты односолитонного решения можно представить в виде

$$
\begin{aligned}
& q_{1 j}(x, t)=-2\left(\lambda_{1}^{+}+\left(\lambda_{1}^{+}\right)^{*}\right) \frac{n_{0,1}^{1}\left(n_{0,1}^{j}\right)^{*} e^{-i\left(\widetilde{M}_{1}^{+}(x, t)+\left(\widetilde{M}_{1}^{+}\right)^{*}(-x, t)\right)}}{2 R_{0,1} \operatorname{ch}\left(2 \nu_{1} x+2 \tilde{\mu}_{1} t+\xi_{0,1}\right)}, \\
& q_{j 1}(x, t)=-2\left(\lambda_{1}^{+}+\left(\lambda_{1}^{+}\right)^{*}\right) \frac{n_{0,1}^{j}\left(n_{0,1}^{1}\right)^{*} e^{i\left(\widetilde{M}_{1}^{+}(x, t)+\left(\widetilde{M}_{1}^{+}\right)^{*}(-x, t)\right)}}{2 R_{0,1} \operatorname{ch}\left(2 \nu_{1} x+2 \tilde{\mu}_{1} t+\xi_{0,1}\right)}
\end{aligned}
$$

где

$$
\begin{aligned}
\widetilde{M}_{1}^{ \pm}(x, t) & =\lambda_{1}^{ \pm} x+2 f_{0,1}^{ \pm} t, \quad\left(\widetilde{M}_{1}^{+}(-x, t)\right)^{*}=\left(-\lambda_{1}^{+}\right)^{*}(-x)+2\left(f_{0}\left(-\lambda_{1}^{+}\right)^{*}\right) t \\
\nu_{1} & =\frac{i\left(\left(-\lambda_{1}^{+}\right)^{*}-\lambda_{1}^{+}\right)}{2}, \quad \tilde{\mu}_{1}=i\left(f\left(-\lambda_{1}^{+}\right)^{*}-f\left(\lambda_{1}^{+}\right)\right), \\
R_{0,1} & =\sqrt{\left(n_{0,1}^{1}\right)^{*} n_{0,1}^{1}\left(\left(n_{0,1}^{2}\right)^{*} n_{0,1}^{2}+\left(n_{0,1}^{3}\right)^{*} n_{0,1}^{3}\right)} \\
\xi_{0,1} & =\frac{1}{2} \ln \frac{\left(n_{0,1}^{1}\right)^{*} n_{0,1}^{1}}{\left(n_{0,1}^{2}\right)^{*} n_{0,1}^{2}+\left(n_{0,1}^{3}\right)^{*} n_{0,1}^{3}}
\end{aligned}
$$


ПримеР 4. Если снова взять $\mathfrak{g} \simeq s l(3, \mathbb{C})$ и автоморфизм инволюции, который является отражением Вейля относительно второго простого корня $\mathrm{e}_{2}-\mathrm{e}_{3}$ алгебры $s l(3, \mathbb{C})$, и применить редукцию типа $(\mathrm{B})(16)$, то получим еще одну блочную матрицу $B=\left(\begin{array}{cc}\mathbf{B}_{+} & 0 \\ 0 & \mathbf{B}_{-}\end{array}\right)$, где $\mathbf{B}_{-}-$блочная внедиагональная матрица. Блоки можно представить следующим образом:

$$
\mathbf{B}_{+}=\mp 1, \quad \mathbf{B}_{-}=\left(\begin{array}{cc}
0 & \pm 1 \\
\pm 1 & 0
\end{array}\right)
$$

Условия редукции для матричных блоков имеют вид

$$
(C): \begin{aligned}
& \mathbf{q}^{-}(x, t)=-\mathbf{B}_{-}\left(\mathbf{q}^{+}(-x, t)\right)^{\dagger}\left(\mathbf{B}_{+}\right)^{-1}, \\
& \mathbf{q}^{+}(x, t)=-\mathbf{B}_{+}\left(\mathbf{q}^{-}(-x, t)\right)^{\dagger}\left(\mathbf{B}_{-}\right)^{-1} .
\end{aligned}
$$

Теперь если применить инволюцию типа $(\mathrm{C})$, используя (63), то стандартное векторное обобщение НУШ, предложенное Манаковым, принимает вид

$$
\begin{aligned}
& -i q_{1, t}+q_{1, x x}+2\left(q_{1}(x, t) q_{2}^{*}(-x, t)+q_{2}(x, t) q_{1}^{*}(-x, t)\right) q_{1}(x, t)=0, \\
& -i q_{2, t}+q_{2, x x}+2\left(q_{1}(x, t) q_{2}^{*}(-x, t)+q_{2}(x, t) q_{1}^{*}(-x, t)\right) q_{2}(x, t)=0 .
\end{aligned}
$$

Также можно представить односолитонное решение векторного обобщения НУШ, предложенного Манаковым, для этого типа инволюции как одевающий множитель (49), удовлетворяющий инволюции (58) с условием (59). Итак, из (54) следует, что компоненты односолитонного решения можно представить в виде

$$
\begin{aligned}
& q_{12}(x, t)=-2\left(\lambda_{1}^{+}+\left(\lambda_{1}^{+}\right)^{*}\right) n_{0,1}^{1} e^{-i \widetilde{M}_{1}^{+}(x, t)} R_{1}^{-1}(x, t)\left(n_{0,1}^{3}\right)^{*} e^{-i \widetilde{M}_{1}^{+, *}(-x, t)}, \\
& q_{13}(x, t)=-2\left(\lambda_{1}^{+}+\left(\lambda_{1}^{+}\right)^{*}\right) n_{0,1}^{1} e^{-i \widetilde{M}_{1}^{+}(x, t)} R_{1}^{-1}(x, t)\left(n_{0,1}^{2}\right)^{*} e^{-i \widetilde{M}_{1}^{+, *}(-x, t)}, \\
& q_{21}(x, t)=-2\left(\lambda_{1}^{+}+\left(\lambda_{1}^{+}\right)^{*}\right) n_{0,1}^{2} e^{i \widetilde{M}_{1}^{+}(x, t)} R_{1}^{-1}(x, t)\left(n_{0,1}^{1}\right)^{*} e^{i \widetilde{M}_{1}^{+, *}(-x, t)}, \\
& q_{31}(x, t)=-2\left(\lambda_{1}^{+}+\left(\lambda_{1}^{+}\right)^{*}\right) n_{0,1}^{3} e^{i \widetilde{M}_{1}^{+}(x, t)} R_{1}^{-1}(x, t)\left(n_{0,1}^{1}\right)^{*} e^{i \widetilde{M}_{1}^{+, *}(-x, t)},
\end{aligned}
$$

где

$$
\begin{gathered}
\widetilde{M}_{1}^{ \pm}(x, t)=\lambda_{1}^{ \pm} x+2 f_{0,1}^{ \pm} t, \quad\left(\widetilde{M}_{1}^{+}(-x, t)\right)^{*}=\left(-\lambda_{1}^{+}\right)^{*}(-x)+2\left(f_{0}\left(-\lambda_{1}^{+}\right)^{*}\right) t, \\
R_{1}(x, t)=2 R_{0,1} \operatorname{ch}\left(2 \nu_{1} x+2 \tilde{\mu}_{1} t+\xi_{0,1}\right), \\
\nu_{1}=\frac{i\left(\left(-\lambda_{1}^{+}\right)^{*}-\lambda_{1}^{+}\right)}{2}, \quad \tilde{\mu}_{1}=i\left(f\left(-\lambda_{1}^{+}\right)^{*}-f\left(\lambda_{1}^{+}\right)\right), \\
R_{0,1}=\sqrt{\left(n_{0,1}^{1}\right)^{*} n_{0,1}^{1}\left(\left(n_{0,1}^{3}\right)^{*} n_{0,1}^{2}+\left(n_{0,1}^{2}\right)^{*} n_{0,1}^{3}\right)}, \\
\xi_{0,1}=\frac{1}{2} \ln \frac{\left(n_{0,1}^{1}\right)^{*} n_{0,1}^{1}}{\left(n_{0,1}^{3}\right)^{*} n_{0,1}^{2}+\left(n_{0,1}^{2}\right)^{*} n_{0,1}^{3}} .
\end{gathered}
$$

ПримеР 5 . Если взять $\mathfrak{g} \simeq s o(5, \mathbb{C})$, то одевающий множитель примет вид $(56)$. Применим нелокальные условия редукции (16),

$$
B u_{1}\left(-x, t,-\lambda^{*}\right)^{\dagger} B^{-1}=u_{1}^{-1}(x, t, \lambda),
$$


и получим следующее условие редукции:

$$
P_{1}(x, t)=B P_{1}^{\dagger}(-x, t) B^{-1}, \quad\left(-\lambda^{ \pm}\right)^{*}=\lambda^{\mp} .
$$

Это значит, что проектор $P_{1}(x, t)$ и $c_{1}(\lambda)$ имеют вид

$$
\begin{gathered}
P_{1}(x, t)=\frac{\left|n_{1}(x, t)\right\rangle\left\langle n_{1}^{*}(-x, t)\right| B}{\left\langle n_{1}^{*}(-x, t)|B| n_{1}(x, t)\right\rangle}, \quad\left\langle m_{1}(x, t)\right|=\left(B\left|n_{1}(-x, t)\right\rangle\right)^{\dagger}, \\
c_{1}(\lambda)=\frac{\lambda-\lambda_{1}^{+}}{\lambda+\left(\lambda_{1}^{+}\right)^{*}} .
\end{gathered}
$$

Таким образом, выбирая тривиальное решение $Q_{0}(x, t)=0$, можно представить компоненты односолитонного решения в виде

$$
\begin{aligned}
q_{12}^{+}(x, t) & =q_{45}^{+}(x, t)= \\
& =-\left(\lambda_{1}^{+}+\left(\lambda_{1}^{+}\right)^{*}\right) R_{1}^{-1}(x, t)\left(n_{0,1}^{1}\left(n_{0,1}^{2}\right)^{*} e^{-i M_{1}^{+}(x, t)}-n_{0,1}^{4}\left(n_{0,1}^{5}\right)^{*} e^{-i\left(M_{1}^{+}(-x, t)\right)^{*}}\right), \\
q_{13}^{+}(x, t) & =-q_{35}^{+}(x, t)= \\
& =-\left(\lambda_{1}^{+}+\left(\lambda_{1}^{+}\right)^{*}\right) R_{1}^{-1}(x, t)\left(n_{0,1}^{1}\left(n_{0,1}^{3}\right)^{*} e^{-i M_{1}^{+}(x, t)}+n_{0,1}^{3}\left(n_{0,1}^{5}\right)^{*} e^{-i\left(M_{1}^{+}(-x, t)\right)^{*}}\right), \\
q_{14}^{+}(x, t) & =q_{25}^{+}(x, t)= \\
& =-\left(\lambda_{1}^{+}+\left(\lambda_{1}^{+}\right)^{*}\right) R_{1}^{-1}(x, t)\left(n_{0,1}^{1}\left(n_{0,1}^{4}\right)^{*} e^{-i M_{1}^{+}(x, t)}-n_{0,1}^{2}\left(n_{0,1}^{5}\right)^{*} e^{-i\left(M_{1}^{+}(-x, t)\right)^{*}}\right), \\
q_{21}^{-}(x, t) & =q_{54}^{-}(x, t)= \\
& =-\left(\lambda_{1}^{+}+\left(\lambda_{1}^{+}\right)^{*}\right) R_{1}^{-1}(x, t)\left(n_{0,1}^{2}\left(n_{0,1}^{1}\right)^{*} e^{i\left(M_{1}^{-}(-x, t)\right)^{*}}-n_{0,1}^{5}\left(n_{0,1}^{4}\right)^{*} e^{i M_{1}^{+}(x, t)}\right), \\
q_{31}^{-}(x, t) & =-q_{53}^{-}(x, t)= \\
& =-\left(\lambda_{1}^{+}+\left(\lambda_{1}^{+}\right)^{*}\right) R_{1}^{-1}(x, t)\left(n_{0,1}^{3}\left(n_{0,1}^{1}\right)^{*} e^{i\left(M_{1}^{-}(-x, t)\right)^{*}}+n_{0,1}^{5}\left(n_{0,1}^{3}\right)^{*} e^{i M_{1}^{+}(x, t)}\right), \\
q_{41}^{-}(x, t) & =q_{52}^{-}(x, t)= \\
& =-\left(\lambda_{1}^{+}+\left(\lambda_{1}^{+}\right)^{*}\right) R_{1}^{-1}(x, t)\left(n_{0,1}^{4}\left(n_{0,1}^{1}\right)^{*} e^{i\left(M_{1}^{+}(-x, t)\right)^{*}}-n_{0,1}^{5}\left(n_{01}^{2}\right)^{*} e^{i M_{1}^{+}(x, t)}\right),
\end{aligned}
$$

где

$$
\begin{gathered}
\left(M_{1}^{+}(-x, t)\right)^{*}=\left(-\lambda_{1}^{+}\right)^{*}(-x)+2\left(f\left(-\lambda_{1}^{+}\right)^{*}\right) t, \\
R_{1}(x, t)=2 R_{0,1} \operatorname{ch}\left(2 \nu_{1} x+2 \tilde{\mu}_{1} t+\xi_{0,1}\right)+S, \\
\nu_{1}=\frac{i\left(\left(-\lambda_{1}^{+}\right)^{*}-\lambda_{1}^{+}\right)}{2}, \quad \tilde{\mu}_{1}=i\left(f\left(-\lambda_{1}^{+}\right)^{*}-f\left(\lambda_{1}^{+}\right)\right), \\
S=\left(n_{0,1}^{2}\right)^{*} n_{0,1}^{2}+\left(n_{0,1}^{3}\right)^{*} n_{0,1}^{3}+\left(n_{0,1}^{4}\right)^{*} n_{0,1}^{4}, \\
R_{0,1}=\sqrt{\left(n_{0,1}^{1}\right)^{*} n_{0,1}^{1}+\left(n_{0,1}^{5}\right)^{*} n_{0,1}^{5}}, \quad \xi_{0,1}=\frac{1}{2} \ln \frac{\left(n_{0,1}^{1}\right)^{*} n_{0,1}^{1}}{\left(n_{0,1}^{5}\right)^{*} n_{0,1}^{5}} .
\end{gathered}
$$

Пример 6. Снова возьмем $\mathfrak{g} \simeq s o(5, \mathbb{C})$ и другую $\mathbb{Z}_{2}$-редукцию типа (В) (16) с блочной матрицей $B$, имеющей внедиагональный блок $\mathbf{B}_{ \pm}: \mathbf{B}_{+}=\mathbf{B}_{-}=\mp 1$ и $\mathbf{B}_{ \pm}=$off-diag $( \pm 1, \ldots, \pm 1)$. В этом случае условия редукции для матричных блоков имеют вид

$$
\mathbf{q}^{-}(x, t)=-\mathbf{B}_{-}\left(\mathbf{q}^{+}(-x, t)\right)^{\dagger}\left(\mathbf{B}_{+}\right)^{-1}, \quad \mathbf{q}^{+}(x, t)=-\mathbf{B}_{+}\left(\mathbf{q}^{-}(-x, t)\right)^{\dagger}\left(\mathbf{B}_{-}\right)^{-1} .
$$


Теперь вспомним пример 1 из раздела 2. Если применить инволюцию типа (В), используя $\mathbf{B}_{+}=\mathbf{B}_{-}=\mp 1$ и $\mathbf{B}_{ \pm}=\operatorname{off}-\operatorname{diag}( \pm 1, \ldots, \pm 1)$, то трехкомпонентное НУШ (24) можно представить в виде

$$
\begin{aligned}
& i\left(q_{t}^{+}\right)_{12}+\left(q_{x x}^{+}\right)_{12}+2\left(q_{12}(x, t) q_{14}^{*}(-x, t)+\right. \\
& \left.\quad+2 q_{13}(x, t) q_{13}^{*}(-x, t)\right) q_{12}+2 q_{12}^{+, *}(-x, t)\left(q_{13}^{+}\right)^{2}=0, \\
& i\left(q_{t}^{+}\right)_{13}+\left(q_{x x}^{+}\right)_{13}+2\left(q_{12}(x, t) q_{14}^{*}(-x, t)+q_{13}(x, t) q_{13}^{*}(-x, t)+\right. \\
& \left.\quad+q_{14}(x, t) q_{12}^{*}(-x, t)\right) q_{13}+2 q_{13}^{+, *}(-x, t) q_{14}^{+} q_{12}^{+}=0, \\
& \quad i\left(q_{t}^{+}\right)_{14}+\left(q_{x x}^{+}\right)_{14}+2\left(q_{14}(x, t) q_{12}^{*}(-x, t)+\right. \\
& \left.\quad+2 q_{13}(x, t) q_{13}^{*}(-x, t)\right) q_{14}+2 q_{14}^{+, *}(-x, t)\left(q_{13}^{+}\right)^{2}=0 .
\end{aligned}
$$

Итак, односолитонное решение этой системы для этого типа инволюции можно записать как одевающий множитель (56), удовлетворяющий инволюции (68) с условием (69). Из (57) следует, что компоненты односолитонного решения можно представить в виде

$$
\begin{aligned}
q_{12}^{+}(x, t)= & q_{45}^{+}(x, t)=-\left(\lambda_{1}^{+}+\left(\lambda_{1}^{+}\right)^{*}\right) R_{1}^{-1}(x, t) \times \\
& \times\left(n_{0,1}^{1}\left(n_{0,1}^{4}\right)^{*} e^{-i M_{1}^{+}(x, t)}-n_{0,1}^{4}\left(n_{0,1}^{5}\right)^{*} e^{-i\left(M_{1}^{+}(-x, t)\right)^{*}}\right), \\
q_{13}^{+}(x, t)= & -q_{35}^{+}(x, t)=-\left(\lambda_{1}^{+}+\left(\lambda_{1}^{+}\right)^{*}\right) R_{1}^{-1}(x, t) \times \\
& \times\left(n_{0,1}^{1}\left(n_{0,1}^{3}\right)^{*} e^{-i M_{1}^{+}(x, t)}+n_{0,1}^{3}\left(n_{0,1}^{5}\right)^{*} e^{-i\left(M_{1}^{+}(-x, t)\right)^{*}}\right), \\
q_{14}^{+}(x, t)= & q_{25}^{+}(x, t)=-\left(\lambda_{1}^{+}+\left(\lambda_{1}^{+}\right)^{*}\right) R_{1}^{-1}(x, t) \times \\
& \times\left(n_{0,1}^{1}\left(n_{0,1}^{2}\right)^{*} e^{-i M_{1}^{+}(x, t)}-n_{0,1}^{2}\left(n_{0,1}^{5}\right)^{*} e^{-i\left(M_{1}^{+}(-x, t)\right)^{*}}\right), \\
q_{21}^{-}(x, t)= & q_{54}^{-}(x, t)=-\left(\lambda_{1}^{+}+\left(\lambda_{1}^{+}\right)^{*}\right) R_{1}^{-1}(x, t) \times \\
& \times\left(n_{0,1}^{2}\left(n_{0,1}^{1}\right)^{*} e^{i\left(M_{1}^{-}(-x, t)\right)^{*}}-n_{0,1}^{5}\left(n_{0,1}^{2}\right)^{*} e^{i M_{1}^{+}(x, t)}\right), \\
q_{31}^{-}(x, t)= & -q_{53}^{-}(x, t)=-\left(\lambda_{1}^{+}+\left(\lambda_{1}^{+}\right)^{*}\right) R_{1}^{-1}(x, t) \times \\
& \times\left(n_{0,1}^{3}\left(n_{0,1}^{1}\right)^{*} e^{i\left(M_{1}^{-}(-x, t)\right)^{*}}+n_{0,1}^{5}\left(n_{0,1}^{3}\right)^{*} e^{i M_{1}^{+}(x, t)}\right), \\
q_{41}^{-}(x, t)= & q_{52}^{-}(x, t)=-\left(\lambda_{1}^{+}+\left(\lambda_{1}^{+}\right)^{*}\right) R_{1}^{-1}(x, t) \times \\
& \times\left(n_{0,1}^{4}\left(n_{0,1}^{1}\right)^{*} e^{i\left(M_{1}^{+}(-x, t)\right)^{*}}-n_{0,1}^{5}\left(n_{01}^{4}\right)^{*} e^{i M_{1}^{+}(x, t)}\right),
\end{aligned}
$$

где

$$
\begin{gathered}
\left(M_{1}^{+}(-x, t)\right)^{*}=\left(-\lambda_{1}^{+}\right)^{*}(-x)+2\left(f\left(-\lambda_{1}^{+}\right)^{*}\right) t, \\
R_{1}(x, t)=2 R_{0,1} \operatorname{ch}\left(2 \nu_{1} x+2 \tilde{\mu}_{1} t+\xi_{0,1}\right)+S, \\
\nu_{1}=\frac{i\left(\left(-\lambda_{1}^{+}\right)^{*}-\lambda_{1}^{+}\right)}{2}, \quad \tilde{\mu}_{1}=i\left(f\left(-\lambda_{1}^{+}\right)^{*}-f\left(\lambda_{1}^{+}\right)\right), \\
S=\left(n_{0,1}^{4}\right)^{*} n_{0,1}^{2}+\left(n_{0,1}^{3}\right)^{*} n_{0,1}^{3}+\left(n_{0,1}^{2}\right)^{*} n_{0,1}^{4}, \\
R_{0,1}=\sqrt{\left(n_{0,1}^{1}\right)^{*} n_{0,1}^{1}+\left(n_{0,1}^{5}\right)^{*} n_{0,1}^{5}}, \quad \xi_{0,1}=\frac{1}{2} \ln \frac{\left(n_{0,1}^{1}\right)^{*} n_{0,1}^{1}}{\left(n_{0,1}^{5}\right)^{*} n_{0,1}^{5}} .
\end{gathered}
$$




\section{3. Двухсолитонное решение.}

ПримеР 7 . При $\mathfrak{g} \simeq \operatorname{sl}(3, \mathbb{C})$, для того чтобы получить двухсолитонное решение, запишем одевающий множитель (47) в виде

$$
\begin{aligned}
\eta^{+}(x, t, \lambda) & =u_{1,2}(x, t, \lambda) \eta_{0}^{+}(x, t, \lambda)\left(\omega_{1,2}^{ \pm}(\lambda)\right)^{-1}, \\
u_{1,2}(x, t, \lambda) & =\mathbb{1}+\left(c_{1}(\lambda)-1\right) P_{1}(x, t)+\left(c_{2}(\lambda)-1\right) P_{2}(x, t) .
\end{aligned}
$$

Таким образом, сингулярное решение (двухсолитонное решение) с сингулярностями в точках $\lambda_{1}^{ \pm}$и $\lambda_{2}^{ \pm}$можно получить следующим образом:

$$
Q(x, t)=Q_{0}(x, t)-\sum_{k=1}^{2}\left(\lambda_{k}^{+}-\lambda_{k}^{-}\right)\left[\mathrm{J}, P_{k}(x, t)\right]
$$

В случае канонической редукции (16) типа (В) при $B=\mathbb{I}$ из (78) следует, что компоненты двухсолитонного решения можно представить в виде

$$
\begin{array}{ll}
q_{1 j}(x, t)=-2 \sum_{k=1}^{2}\left(\lambda_{k}^{+}+\left(\lambda_{k}^{+}\right)^{*}\right) \frac{n_{0, k}^{k}\left(n_{0, k}^{j}\right)^{*} e^{-i\left(\widetilde{M}_{k}^{+}(x, t)+\left(\widetilde{M}_{k}^{+}\right)^{*}(-x, t)\right)}}{2 R_{0, k} \operatorname{ch}\left(2 \nu_{k} x+2 \tilde{\mu}_{k} t+\xi_{0, k}\right)}, & j=2,3, \\
q_{j 1}(x, t)=2 \sum_{k=1}^{2}\left(\lambda_{k}^{+}+\left(\lambda_{k}^{+}\right)^{*}\right) \frac{n_{0, k}^{j}\left(n_{0, k}^{1}\right)^{*} e^{i\left(\widetilde{M}_{k}^{+}(x, t)+\left(\widetilde{M}_{k}^{+}\right)^{*}(-x, t)\right)}}{2 R_{0, k} \operatorname{ch}\left(2 \nu_{k} x+2 \tilde{\mu}_{k} t+\xi_{0, k}\right)}, & j=2,3,
\end{array}
$$

где

$$
\begin{gathered}
\widetilde{M}_{k}^{ \pm}(x, t)=\lambda_{k}^{ \pm} x+2 f_{0, k}^{ \pm} t, \quad\left(\widetilde{M}_{k}^{+}(-x, t)\right)^{*}=\left(-\lambda_{k}^{+}\right)^{*}(-x)+2\left(f\left(-\lambda_{1}^{+}\right)^{*}\right) t, \\
\nu_{k}=\frac{i\left(\left(-\lambda_{k}^{+}\right)^{*}-\lambda_{k}^{+}\right)}{2}, \quad \tilde{\mu}_{k}=i\left(f\left(-\lambda_{k}^{+}\right)^{*}-f\left(\lambda_{k}^{+}\right)\right), \\
R_{0, k}=\sqrt{\left(n_{0, k}^{1}\right)^{*} n_{0, k}^{1}\left(\left(n_{0, k}^{2}\right)^{*} n_{0, k}^{2}+\left(n_{0, k}^{3}\right)^{*} n_{0, k}^{3}\right)}, \\
\xi_{0, k}=\frac{1}{2} \ln \frac{\left(n_{0, k}^{1}\right)^{*} n_{0, k}^{1}}{\left(n_{0, k}^{2}\right)^{*} n_{0, k}^{2}+\left(n_{0, k}^{3}\right)^{*} n_{0, k}^{3}} .
\end{gathered}
$$

ЗАмЕЧАниЕ 1. Можно получить двухсолитонное решение другим способом, а именно применить метод одевания снова к односолитонному решению, которое используется как затравочное решение:

$$
\eta^{+}(x, t, \lambda)=u_{2}(x, t, \lambda) \eta_{1}^{+}(x, t, \lambda)\left(\omega_{2}^{ \pm}(\lambda)\right)^{-1}, \quad u_{2}(x, t, \lambda)=\mathbb{1}+\left(c_{2}(\lambda)-1\right) P_{2}(x, t) .
$$


ПримеР 8. В случае инволюции из примера 4 двухсолитонный одевающий множитель (77а) является автоматически совместным с инволюцией. Следовательно, в силу (78) вытекает, что компоненты двухсолитонного решения можно представить как

$$
\begin{aligned}
& q_{12}(x, t)=-2 \sum_{k=1}^{2}\left(\lambda_{k}^{+}+\left(\lambda_{k}^{+}\right)^{*}\right) n_{0, k}^{1} e^{-i \widetilde{M}_{k}^{+}(x, t)} R_{k}^{-1}(x, t)\left(n_{0, k}^{3}\right)^{*} e^{-i \widetilde{M}_{k}^{+, *}(-x, t)}, \\
& q_{13}(x, t)=-2 \sum_{k=1}^{2}\left(\lambda_{k}^{+}+\left(\lambda_{k}^{+}\right)^{*}\right) n_{0, k}^{1} e^{-i \widetilde{M}_{k}^{+}(x, t)} R_{k}^{-1}(x, t)\left(n_{0, k}^{2}\right)^{*} e^{-i \widetilde{M}_{k}^{+, *}(-x, t)}, \\
& q_{21}(x, t)=-2 \sum_{k=1}^{2}\left(\lambda_{k}^{+}+\left(\lambda_{k}^{+}\right)^{*}\right) n_{0, k}^{2} e^{i \widetilde{M}_{k}^{+}(x, t)} R_{k}^{-1}(x, t)\left(n_{0, k}^{1}\right)^{*} e^{i \widetilde{M}_{k}^{+, *}(-x, t)}, \\
& q_{31}(x, t)=-2 \sum_{k=1}^{2}\left(\lambda_{k}^{+}+\left(\lambda_{k}^{+}\right)^{*}\right) n_{0, k}^{3} e^{i \widetilde{M}_{k}^{+}(x, t)} R_{k}^{-1}(x, t)\left(n_{0, k}^{1}\right)^{*} e^{i \widetilde{M}_{k}^{+, *}(-x, t)},
\end{aligned}
$$

где

$$
\begin{gathered}
\widetilde{M}_{k}^{ \pm}(x, t)=\lambda_{k}^{ \pm} x+2 f_{0, k}^{ \pm} t, \quad\left(\widetilde{M}_{k}^{+}(-x, t)\right)^{*}=\left(-\lambda_{k}^{+}\right)^{*}(-x)+2\left(f\left(-\lambda_{1}^{+}\right)^{*}\right) t, \\
R_{1}(x, t)=2 R_{0,1} \operatorname{ch}\left(2 \nu_{1} x+2 \tilde{\mu}_{1} t+\xi_{0,1}\right), \\
\nu_{k}=\frac{i\left(\left(-\lambda_{k}^{+}\right)^{*}-\lambda_{k}^{+}\right)}{2}, \quad \tilde{\mu}_{k}=i\left(f\left(-\lambda_{k}^{+}\right)^{*}-f\left(\lambda_{k}^{+}\right)\right), \\
R_{0, k}=\sqrt{\left(n_{0, k}^{1}\right)^{*} n_{0, k}^{1}\left(\left(n_{0, k}^{3}\right)^{*} n_{0, k}^{2}+\left(n_{0, k}^{2}\right)^{*} n_{0, k}^{3}\right)}, \\
\xi_{0, k}=\frac{1}{2} \ln \frac{\left(n_{0, k}^{1}\right)^{*} n_{0, k}^{1}}{\left(n_{0, k}^{3}\right)^{*} n_{0, k}^{2}+\left(n_{0, k}^{2}\right)^{*} n_{0, k}^{3}} .
\end{gathered}
$$

ПримеР 9. Если взять $\mathfrak{g} \simeq s o(5, \mathbb{C})$, то двухсолитонные одевающие множители $u(x, t, \lambda)$ можно рассматривать с двумя дополнительными полюсами в виде

$$
\begin{aligned}
& u_{1,2}(x, t, \lambda)=\mathbb{1}+\left(c_{1}(\lambda)-1\right) P_{1}(x, t)+\left(\frac{1}{c_{1}(\lambda)}-1\right) \bar{P}_{1}(x, t)+ \\
&+\left(c_{2}(\lambda)-1\right) P_{2}(x, t)+\left(\frac{1}{c_{2}(\lambda)}-1\right) \bar{P}_{2}(x, t), \\
& c_{k}(\lambda)=\frac{\lambda-\lambda_{k}^{+}}{\lambda-\lambda_{k}^{-}}, \quad P_{k}(x, t)=\frac{\left|n_{k}(x, t)\right\rangle\left\langle m_{k}(x, t)\right|}{\left\langle m_{k}(x, t) \mid n_{k}(x, t)\right\rangle}, \\
& \bar{P}_{k}=S_{0} P_{k}^{\mathrm{T}} S_{0}^{-1}, \quad k=1,2 .
\end{aligned}
$$

Таким образом, новый потенциал $Q(x, t)$, соответствующий данному тривиальному решению $Q_{0}(x, t)=0$, можно получить в виде

$$
Q(x, t)=-\sum_{k=1}^{2}\left(\lambda_{k}^{+}-\lambda_{k}^{-}\right)\left[J, P_{k}(x, t)-\bar{P}_{k}(x, t)\right] .
$$


Компоненты нового потенциала $Q(x, t)$ имеют вид

$$
\begin{aligned}
& q_{12}^{+}(x, t)= q_{45}^{+}(x, t)=-\sum_{k=1}^{2}\left(\lambda_{k}^{+}-\lambda_{k}^{-}\right) R_{k}^{-1}(x, t) \times \\
& \times\left(n_{0, k}^{1} m_{0, k}^{2} e^{-i M_{k}^{+}(x, t)}+n_{0, k}^{4} m_{0, k}^{5} e^{-i M_{k}^{-}(x, t)}\right), \\
& q_{13}^{+}(x, t)=-q_{35}^{+}(x, t)=-\sum_{k=1}^{2}\left(\lambda_{k}^{+}-\lambda_{k}^{-}\right) R_{k}^{-1}(x, t) \times \\
& \times\left(n_{0, k}^{1} m_{0, k}^{3} e^{-i M_{k}^{+}(x, t)}-n_{0, k}^{3} m_{0, k}^{5} e^{-i M_{k}^{-}(x, t)}\right), \\
& q_{14}^{+}(x, t)= q_{25}^{+}(x, t)=-\sum_{k=1}^{2}\left(\lambda_{k}^{+}-\lambda_{k}^{-}\right) R_{k}^{-1}(x, t) \times \\
& \times\left(n_{0, k}^{1} m_{0, k}^{4} e^{-i M_{k}^{+}(x, t)}+n_{0, k}^{2} m_{0, k}^{5} e^{-i M_{k}^{-}(x, t)}\right), \\
& q_{21}^{-}(x, t)= q_{54}^{-}(x, t)=-\sum_{k=1}^{2}\left(\lambda_{k}^{+}-\lambda_{k}^{-}\right) R_{k}^{-1}(x, t) \times \\
& \times\left(-n_{0, k}^{2} m_{0, k}^{1} e^{i M_{k}^{-}(x, t)}-n_{0, k}^{5} m_{0, k}^{4} e^{i M_{k}^{+}(x, t)}\right), \\
& \times\left(-n_{0, k}^{4} m_{0, k}^{1} e^{i M_{k}^{-}(x, t)}-n_{0, k}^{5} m_{0, k}^{2} e^{i M_{k}^{+}(x, t)}\right), \\
& \times\left(-n_{0, k}^{3} m_{0, k}^{1} e^{i M_{k}^{-}(x, t)}+n_{0, k}^{5} m_{0, k}^{3} e^{i M_{k}^{+}(x, t)}\right), \\
& q_{31}^{-}(x, t)= q_{52}^{-}(x, t)=-\sum_{53}^{2}\left(\lambda_{k}^{+}-\lambda_{k}^{-}\right) R_{k}^{-1}(x, t) \times \\
&\left.q_{k}^{-}(x, t)=-\lambda_{k}^{-}\right) R_{k}^{-1}(x, t) \times \\
& \sum_{k}^{+}(x)=1
\end{aligned}
$$

где

$$
\begin{gathered}
M_{k}^{ \pm}(x, t)=\lambda_{k}^{ \pm} x+2 f_{0, k}^{ \pm} t, \quad R_{k}(x, t)=2 R_{0, k} \operatorname{ch}\left(2 \nu_{k} x+2 \tilde{\mu}_{k} t+\xi_{0, k}\right)+S, \\
\nu_{k}=\frac{i\left(\lambda_{k}^{-}-\lambda_{k}^{+}\right)}{2}, \quad \tilde{\mu}_{k}=i\left(f\left(\lambda_{k}^{-}\right)-f\left(\lambda_{k}^{+}\right)\right), \\
S=m_{0, k}^{2} n_{0, k}^{2}+m_{0, k}^{3} n_{0, k}^{3}+m_{0, k}^{4} n_{0, k}^{4}, \\
R_{0, k}=\sqrt{m_{0, k}^{1} n_{0, k}^{1}+m_{0, k}^{5} n_{0, k}^{5}}, \quad \xi_{0, k}=\frac{1}{2} \ln \frac{m_{0, k}^{1} n_{0, k}^{1}}{m_{0, k}^{5} n_{0, k}^{5}} .
\end{gathered}
$$

ПримеР 10. Снова рассмотрим случай, когда $\mathfrak{g} \simeq s o(5, \mathbb{C})$. Если применить нелокальную инволюцию вида (16), то одевающий множитель (84) будет удовлетворять следующему условию редукции:

$$
B \prod_{k=1}^{2} u_{k}\left(-x, t,-\lambda^{*}\right)^{\dagger} B^{-1}=\prod_{k=1}^{2} u_{k}^{-1}(x, t, \lambda),
$$

где $B$ - постоянная блочно-диагональная матрица. Эта инволюция имеет место, если

$$
P_{k}(x, t)=B P_{k}^{\dagger}(-x, t) B^{-1}, \quad\left(-\lambda^{ \pm}\right)^{*}=\lambda^{\mp} .
$$


Таким образом, проектор $P_{k}(x, t)$ и $c_{k}(\lambda)$ принимают вид

$$
\begin{gathered}
P_{k}(x, t)=\frac{\left|n_{k}(x, t)\right\rangle\left\langle n_{k}^{*}(-x, t)\right| B}{\left\langle n_{k}^{*}(-x, t)|B| n_{k}(x, t)\right\rangle}, \quad\left\langle m_{k}(x, t)\right|=\left(B\left|n_{k}(-x, t)\right\rangle\right)^{\dagger}, \\
c_{k}(\lambda)=\frac{\lambda-\lambda_{k}^{+}}{\lambda+\left(\lambda_{k}^{+}\right)^{*}} .
\end{gathered}
$$

Итак, из (57) следует, что

$$
Q(x, t)=Q_{0}(x, t)-\left(\lambda_{k}^{+}+\left(\lambda_{k}^{+}\right)^{*}\right)\left[\mathrm{J}, B P_{k}^{\dagger}(-x, t) B^{-1}\right] .
$$

Значит, компоненты двухсолитонного решения можно представить в виде

$$
\begin{aligned}
& q_{12}^{+}(x, t)=q_{45}^{+}(x, t)=-\sum_{k=1}^{2}\left(\lambda_{k}^{+}+\left(\lambda_{k}^{+}\right)^{*}\right) R_{k}^{-1}(x, t) \times \\
& \times\left(n_{0, k}^{1}\left(n_{0, k}^{2}\right)^{*} e^{-i M_{k}^{+}(x, t)}-n_{0, k}^{4}\left(n_{0, k}^{5}\right)^{*} e^{-i\left(M_{k}^{+}(-x, t)\right)^{*}}\right) \text {, } \\
& q_{13}^{+}(x, t)=-q_{35}^{+}(x, t)=-\sum_{k=1}^{2}\left(\lambda_{k}^{+}+\left(\lambda_{k}^{+}\right)^{*}\right) R_{k}^{-1}(x, t) \times \\
& \times\left(n_{0, k}^{1}\left(n_{0, k}^{3}\right)^{*} e^{-i M_{k}^{+}(x, t)}+n_{0, k}^{3}\left(n_{0, k}^{5}\right)^{*} e^{-i\left(M_{k}^{+}(-x, t)\right)^{*}}\right) \text {, } \\
& q_{14}^{+}(x, t)=q_{25}^{+}(x, t)=-\sum_{k=1}^{2}\left(\lambda_{k}^{+}+\left(\lambda_{k}^{+}\right)^{*}\right) R_{k}^{-1}(x, t) \times \\
& \times\left(n_{0, k}^{1}\left(n_{0, k}^{4}\right)^{*} e^{-i M_{k}^{+}(x, t)}-n_{0, k}^{2}\left(n_{0, k}^{5}\right)^{*} e^{-i\left(M_{k}^{+}(-x, t)\right)^{*}}\right), \\
& q_{21}^{-}(x, t)=q_{54}^{-}(x, t)=-\sum_{k=1}^{2}\left(\lambda_{k}^{+}+\left(\lambda_{k}^{+}\right)^{*}\right) R_{k}^{-1}(x, t) \times \\
& \times\left(n_{0, k}^{2}\left(n_{0, k}^{1}\right)^{*} e^{i\left(M_{k}^{-}(-x, t)\right)^{*}}-n_{0, k}^{5}\left(n_{0, k}^{4}\right)^{*} e^{i M_{k}^{+}(x, t)}\right), \\
& q_{31}^{-}(x, t)=-q_{53}^{-}(x, t)=-\sum_{k=1}^{2}\left(\lambda_{k}^{+}+\left(\lambda_{k}^{+}\right)^{*}\right) R_{k}^{-1}(x, t) \times \\
& \times\left(n_{0, k}^{3}\left(n_{0, k}^{1}\right)^{*} e^{i\left(M_{k}^{-}(-x, t)\right)^{*}}+n_{0, k}^{5}\left(n_{0, k}^{3}\right)^{*} e^{i M_{k}^{+}(x, t)}\right), \\
& q_{41}^{-}(x, t)=q_{52}^{-}(x, t)=-\sum_{k=1}^{2}\left(\lambda_{k}^{+}+\left(\lambda_{k}^{+}\right)^{*}\right) R_{k}^{-1}(x, t) \times \\
& \times\left(n_{0, k}^{4}\left(n_{0, k}^{1}\right)^{*} e^{i\left(M_{k}^{+}(-x, t)\right)^{*}}-n_{0, k}^{5}\left(n_{0, k}^{2}\right)^{*} e^{i M_{k}^{+}(x, t)}\right),
\end{aligned}
$$

где

$$
\begin{aligned}
\left(M_{k}^{+}(-x, t)\right)^{*} & =\left(-\lambda_{k}^{+}\right)^{*}(-x)+2\left(f\left(-\lambda_{1}^{+}\right)^{*}\right) t \\
R_{k}(x, t) & =2 R_{0, k} \operatorname{ch}\left(2 \nu_{k} x+2 \tilde{\mu}_{k} t+\xi_{0, k}\right)+S, \\
\nu_{k} & =\frac{i\left(\left(-\lambda_{k}^{+}\right)^{*}-\lambda_{k}^{+}\right)}{2}, \quad \tilde{\mu}_{k}=i\left(f\left(-\lambda_{k}^{+}\right)^{*}-f\left(\lambda_{k}^{+}\right)\right), \\
S & =\left(n_{0, k}^{2}\right)^{*} n_{0, k}^{2}+\left(n_{0, k}^{3}\right)^{*} n_{0, k}^{3}+\left(n_{0, k}^{4}\right)^{*} n_{0, k}^{4}, \\
R_{0, k} & =\sqrt{\left(n_{0, k}^{1}\right)^{*} n_{0, k}^{1}+\left(n_{0, k}^{5}\right)^{*} n_{0, k}^{5}}, \quad \xi_{0, k}=\frac{1}{2} \ln \frac{\left(n_{0, k}^{1}\right)^{*} n_{0, k}^{1}}{\left(n_{0, k}^{5}\right)^{*} n_{0, k}^{5}} .
\end{aligned}
$$




\section{5. ЗАКЛЮЧЕНИЕ}

Мы рассмотрели многокомпонентные обобщения НУШ в симметрических пространствах типов A.III и BD.I. Наше рассмотрение опирается на основные примеры двух типов: векторное обобщение НУШ, предложенное Манаковым, связанное с симметрическими пространствами типа A.III, и модель Кулиша-Склянина, относящаяся к симметрическим пространствам типа BD.I. Сначала мы сформулировали прямую задачу рассеяния для обеих моделей, т. е. построили решения Йоста, матрицу рассеяния и минимальное множество данных рассеяния. На основе разложения Гаусса для матрицы рассеяния мы также построили фундаментальные аналитические решения.

Оказалось, что спектральные свойства оператора Лакса критическим образом зависят от выбора представления базовой алгебры Ли или симметрического пространства, тогда как минимальное множество данных рассеяния обеспечивается тем же самым множеством функций [43].

Наконец, была представлена некоторая модификация метода одевания и получены одно- и двухсолитонные решения обеих моделей с нелокальными редукциями.

В зависимости от положения дискретных собственных значений $\lambda_{j}^{ \pm}$на спектральной плоскости существуют два режима для двухсолитонного решения. Если два дискретных собственных значения лежат в верхней половине комплексной плоскости, а другие два собственных значения лежат в нижней полуплоскости, то нелокальная инволюция сохраняет их количество внутри каждого контура, это приводит к задаче Римана-Гильберта со сбалансированным числом сингулярностей, и, следовательно, соответствующие двухсолитонные решения регулярны при всех $t$. В противном случае в двухсолитонном решении возникает сингулярность за конечное время, как и в случае, изученном в работах [23]-[25].

Результаты, полученные в данной работе, можно развивать в нескольких направлениях.

1. Разработать калибровочно-ковариантную формулировку многокомпонентных иерархий НУШ в симметрических пространствах, включая соотношения Вронского, “квадраты собственных функций" и их соотношения полноты, описания класса НЭУ, ассоциированных с заданной задачей рассеяния, производящий (рекурсивный) оператор и его спектральное разложение, описание бесконечного множества интегралов движения, иерархии гамильтоновых структур и $r$-матричной формулировки.

2. Изучить калибровочно-эквивалентные системы многокомпонентных ферромагнетиков в симметрических пространствах [51], [55], [56].

3. Изучить различные типы редукций многокомпонентных интегрируемых систем в симметрических пространствах [57]-[59].

4. Изучить ассоциированные преобразования Дарбу и их обобщения для НУШ в эрмитовых симметрических пространствах и получить многосолитонные решения с помощью таких обобщений, включая рациональные решения [54].

5. Обобщить результаты, полученные в этой работе, на случай ненулевых граничных условий (нетривиальный фон) [60], [61]. Этот случай более сложный и требует отдельного рассмотрения.

6. Изучить другие типы интегрируемых иерархий в симметрических пространствах, например квадратичные расслоения (связанные с НУШ с производной потенци- 
ала уравнением Кауфа-Ньюэлла или уравнением Герджикова-Иванова) [4], [62]-[66] или рациональные расслоения [55], [56].

Благодарности. Авторы благодарят профессора Марка Абловица и профессора Владимира Герджикова за многочисленные полезные обсуждения.

\section{Список литературы}

[1] В. Е. Захаров, А. Б. Шабат, "Схема интегрирования нелинейных уравнений математической физики методом обратной задачи рассеяния. I", Функи. анализ и его прил., 8:3 (1974), 43-53; "Интегрирование нелинейных уравнений математической физики методом обратной задачи рассеяния. II", Функи. анализ и его прил., 13:3 (1979), 13-22.

[2] Л. А. Тахтаджян, Л. Д. Фаддеев, Гамильтонов подход в теории солитонов, Наука, М., 1986.

[3] В.Е. Захаров, С.В. Манаков, С. П. Новиков, Л.П. Питаевский, Теория солитонов: метод обратной задачи, Наука, М., 1980.

[4] V.S. Gerdjikov, G. Vilasi, A. B. Yanovski, Integrable Hamiltonian Hierarchies. Spectral and Geometric Methods, Lecture Notes in Physics, 748, Springer, Berlin-Heidelberg, 2008.

[5] M. J. Ablowitz, B. Prinari, A. D. Trubatch, Discrete and Continuous Nonlinear Schrödinger Systems, London Mathematical Society Lecture Note Series, 302, Cambridge Univ. Press, Cambridge, 2004.

[6] R. Beals, R. R. Coifman, "Scattering and inverse scattering for first order systems", Commun. Pure Appl. Math., 37:1 (1984), 39-90; "Scattering and inverse scattering for first order systems: II", Inverse Problems, 3:4 (1987), 577-594.

[7] P. G. Drazin, R. S. Johnson, Solitons: An Introduction, Cambridge Univ. Press, Cambridge, 1989.

[8] V.S. Gerdjikov, "Generalised Fourier transforms for the soliton equations. Gauge-covariant formulation", Inverse Problems, 2 (1986), 51-74.

[9] С. В. Манаков, "К теории двумерной стационарной самофокусировки электромагнитных волн", ЖЭТФ, 65:2 (1973), 505-516.

[10] C. R. Menyuk, "Nonlinear pulse propagation in birefringent optical fibres", IEEE J. Quantum Electron, 23:2 (1987), 174-176.

[11] J. R. Ackerhalt, P. W. Milonni, "Solitons and four-wave mixing", Phys. Rev. A, 33:5 (1986), 3185-3198.

[12] S. Helgason, Differential Geometry, Lie Groups and Symmetric Spaces, Graduate Studies in Mathematics, 34, AMS, Providence, RI, 2001.

[13] R. Gilmore, Lie Groups, Lie Algebras and Some of Their Applications, Dover, Mineola, NY, 2005.

[14] A. P. Fordy, P. P. Kulish, "Nonlinear Schrödinger equations and simple Lie algebras", Commun. Math. Phys., 89:3 (1983), 427-443.

[15] C. Athorne, A. Fordy, "Generalised KdV and MKdV equations associated with symmetric spaces", J. Phys. A: Math. Gen., 20:6 (1987), 1377-1386.

[16] A. P. Fordy, "Derivative nonlinear Schrödinger equations and Hermitian symmetric spaces", J. Phys. A: Math. Gen., 17:6 (1984), 1235-1245.

[17] O. Loos, Symmetric Spaces, v. I: General Theory; v. II: Compact Spaces and Classification, W. A. Benjamin, New York-Amsterdam, 1969.

[18] P. P. Kulish, E. K. Sklyanin, "O $(N)$-invariant nonlinear Schrödinger equation A new completely integrable system", Phys. Lett. A, 84:7 (1981), 349-352.

[19] В. Г. Дринфельд, В. В. Соколов, “Алгебры Ли и уравнения типа Кортевега-де Фриза”, Итоги науки и техн. Сер. Соврем. пробл. матем. Нов. достиж., 24 (1984), 81-180. 
[20] V.S. Gerdjikov, G. G. Grahovski, N. A. Kostov, "Reductions of N-wave interactions related to low-rank simple Lie algebras: I. $\mathbb{Z}_{2}$-reductions", J. Phys. A: Math. Gen., 34:44 (2001), 9425-9461, arXiv: nlin.SI/0006001.

[21] V.S. Gerdjikov, G. G. Grahovski, R. I. Ivanov, N. A. Kostov, " $N$-wave interactions related to simple Lie algebras. $\mathbb{Z}_{2}$-reductions and soliton solutions", Inverse Problems, 17:4 (2001), 999-1015, arXiv: nlin.SI/0009034.

[22] A. V. Mikhailov, "The reduction problem and the inverse scattering problem", Phys. D, 3:1-2 (1981), 73-117.

[23] M. Ablowitz, Z. Musslimani, "Integrable nonlocal nonlinear Schrödinger equation", Phys. Rev. Lett., 110:6 (2013), 064105, 5 pp.

[24] M. Ablowitz, Z. Musslimani, "Integrable discrete $\mathcal{P} \mathcal{T}$ symmetric model", Phys. Rev. E, 90 (2014), 032912, 5 pp.

[25] M. J. Ablowitz, Z. H. Musslimani, "Inverse scattering transform for the integrable nonlocal nonlinear Schrödinger equation", Nonlinearity, 29:3 (2016), 915-946.

[26] V.S. Gerdjikov, A. Saxena, "Complete integrability of nonlocal nonlinear Schrödinger equation", J. Math. Phys., 58:1 (2017), 013502, 33 pp., arXiv: 1510.0480.

[27] V.E. Zakharov, A. V. Mikhailov, "On the integrability of classical spinor models in two-dimensional space-time", Commun. Math. Phys., 74:1 (1980), 21-40.

[28] F. K. Abdullaev, Y.V. Kartashov, V.V. Konotop, D. A. Zezyulin, "Solitons in PT-symmetric nonlinear lattices", Phys. Rev. A, 83:4 (2011), 041805, 4 pp.

[29] I. V. Barashenkov, "Hamiltonian formulation of the standard $\mathcal{P} \mathcal{T}$-symmetric nonlinear Schrödinger dimer", Phys. Rev. A, 90:4 (2014), 045802, 4 pp.

[30] I. V. Barashenkov, D. E. Pelinovsky, P. Dubard, "Dimer with gain and loss: integrability and $\mathcal{P} \mathcal{T}$-symmetry restoration", J. Phys. A: Math. Theor., 48:32 (2015), 325201, 28 pp.

[31] C. E. Rüter, K. G. Makris, R. El-Ganainy, D. N. Christodoulides, M. Segev, D. Kip, "Observation of parity-time symmetry in optics", Nature Phys., 6 (2010), 192-195.

[32] А. А. Зябловский, А. П. Виноградов, А. А. Пухов, А. В. Дорофеенко, А. А. Лисянский, "РТ-симметрия в оптике", УФН, 184:11 (2014), 1177-1198.

[33] C. M. Bender, S. Boettcher, "Real spectra in non-Hermitian Hamiltonians having $\mathcal{P} \mathcal{T}$ symmetry", Phys. Rev. Lett., 80:24 (1998), 5243-5246; C. M. Bender, S. Boettcher,

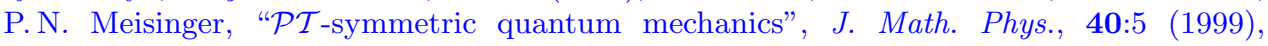
2201-2229.

[34] A. Mostafazadeh, "Pseudo-Hermiticity versus $\mathcal{P} \mathcal{T}$-symmetry: the necessary condition for the reality of the spectrum of a non-Hermitian Hamiltonian", J. Math. Phys., 43:1 (2002), 205-214, arXiv: math-ph/0107001; "Pseudo-Hermiticity versus $\mathcal{P} \mathcal{T}$-symmetry. II. A complete characterization of non-Hermitian Hamiltonians with a real spectrum", J. Math. Phys., 43:1 (2002), 2814-2816, arXiv: math-ph/0110016; "Pseudo-Hermiticity versus $\mathcal{P} \mathcal{T}$-symmetry. III: Equivalence of pseudo-hermiticity and the presence of antilinear symmetries", 45:8 (2002), 3944-3951, arXiv: math-ph/0203005.

[35] A. Mostafazadeh, "Pseudo-Hermiticity and generalized $\mathcal{P} \mathcal{T}$ - and $\mathcal{C P} \mathcal{T}$-symmetries", J. Math. Phys., 44:3 (2003), 974-989, arXiv: math-ph/0209018; "Exact $\mathcal{P} \mathcal{T}$-symmetry is equivalent to Hermiticity", J. Phys. A: Math. Gen., 36:25 (2003), 7081-7091, arXiv: quant-ph/0304080.

[36] C. M. Bender, "Making sense of non-Hermitian Hamiltonians", Rep. Progr. Phys., 70:6 (2007), 947-1018, arXiv: hep-th/0703096.

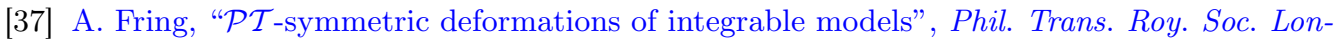
don Ser. A, 371:1989 (2013), 20120046, 18 pp., arXiv: 1204.2291.

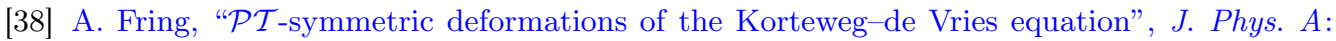
Math. Theor., 40:15 (2007), 4215-4224, arXiv: math-ph/0701036.

[39] V.S. Gerdjikov, "Basic aspects of soliton theory", Proceedings of the Sixth International Conference on Geometry, Integrability and Quantization (Varna, Bulgaria, Sofia, 2-10 June, 2005), eds. I. M. Mladenov, A. C. Hirshfeld, Softex, Sofia, 2005, 78-125. 
[40] V.S. Gerdjikov, D. J. Kaup, N. A. Kostov, T. I. Valchev, "On classification of soliton solutions of multicomponent nonlinear evolution equations", J. Phys. A: Math. Theor., 41:31 (2008), 315213, 36 pp.

[41] V.S. Gerdjikov, "On nonlocal models of Kulish-Sklyanin type and generalized Fourier transforms", Advanced Computing in Industrial Mathematics, Studies in Computational Intelligence, 681, Springer, Cham, 2017, 37-52.

[42] В. С. Герджиков, "Модели типа Кулиша-Склянина: интегрируемость и редукции", TMФ, 192:2 (2017), 187-206, arXiv: 1702.04010.

[43] V.S. Gerdjikov, G. G. Grahovski, "Multi-component NLS models on symmetric spaces: spectral properties versus representations theory", SIGMA, 6 (2010), 044, 29 pp.

[44] V.S. Gerdjikov, "On reductions of soliton solutions of multi-component NLS models and spinor Bose-Einstein condensates", Application of Mathematics in Technical and Natural Sciences, AIP Conference Proceedings, 1186, eds. M. D. Todorov, C. I. Christov, AIP, Melville, NY, 2009, 15-27.

[45] G. G. Grahovski, "On the reductions and scattering data for the generalized Zakharov-Shabat systems", Nonlinear Physics: Theory and Experiment. II, eds. M. J. Ablowitz, M. Boiti, F. Pempinelli, B. Prinari, World Sci., Singapore, 2003, 71-78.

[46] T. I. Valchev, "On Mikhailov's reduction group", Phys. Lett. A, 379:34-35 (2015), $1877-1880$.

[47] V.A. Atanasov, V.S. Gerdjikov, G. G. Grahovski, N. A. Kostov, "Fordy-Kulish model and spinor Bose-Einstein condensate", J. Nonlinear Math. Phys., 15:3 (2008), 291-298.

[48] G. G. Grahovski, V.S. Gerdjikov, N. A. Kostov, "On the multicomponent NLS type equations on symmetric spaces: reductions and soliton solutions", Proceedings of the Sixth International Conference on Geometry, Integrability and Quantization (Varna, Bulgaria, Sofia, 2-10 June, 2005), eds. I. M. Mladenov, A. C. Hirshfeld, Softex, Sofia, 2005, 203-217.

[49] V.S. Gerdjikov, P. P. Kulish, "The generating operator for the $n \times n$ linear system", Phys. D, 3:3 (1981), 549-564.

[50] R. Ivanov, "On the dressing method for the generalised Zakharov-Shabat system", Nucl. Phys. B, 694 (2004), 509-524, arXiv: math-ph/0402031.

[51] V.S. Gerdjikov, G. G. Grahovski, N. A. Kostov, "On $N$-wave type systems and their gauge equivalent", Eur. J. Phys. B, 29:2 (2002), 243-248.

[52] V.S. Gerdjikov, "Algebraic and analytic aspects of $N$-wave type equations", The Legacy of the Inverse Scattering Transform in Applied Mathematics (South Hadley, MA, June 17-21, 2001), Contemporary Mathematics, 301, eds. J. Bona, R. Choudhury, D. Kaup, AMS, Providence, RI, 2002, 35-68, arXiv: nlin.SI/0206014.

[53] А. Б. Шабат, "Обратная задача рассеяния для системы дифференциальных уравнений", Функи. анализ и его прил., 9:3 (1975), 75-78; "Обратная задача рассеяния", Дифферени. уравнения, 15:10 (1979), 1824-1834.

[54] E. V. Doktorov, S. B. Leble, A Dressing Method in Mathematical Physics, Mathematical Physics Studies, 28, Springer, Dordrecht, 2007.

[55] В. С. Герджиков, Г. Г. Граховски, А. В. Михайлов, Т. И. Валчев, "Рациональные пучки и рекурсионные операторы для интегрируемых уравнений на симметричных пространствах типа А.III", ТМФ, 167:3 (2011), 394-406.

[56] V.S. Gerdjikov, G. G. Grahovski, A. V. Mikhailov, T. I. Valchev, "Polynomial bundles and generalised Fourier transforms for integrable equations on A.III-type symmetric spaces", SIGMA, 7 (2011), 096, 48 pp.

[57] G. G. Grahovski, M. Condon, "On the Caudrey-Beals-Coifman system and the gauge group action", J. Nonlin. Math. Phys., 15, suppl. 3 (2008), 197-208, arXiv: 0710.3302.

[58] G. G. Grahovski, V.S. Gerdjikov, N. A. Kostov, V. A. Atanasov, "New integrable multi-component NLS type equations on symmetric spaces: $\mathbb{Z}_{4}$ and $\mathbb{Z}_{6}$ reductions", Proceedings of the Seventh International Conference on Geometry Integrability and Quantization (Varna, Bulgaria), eds. Iv. M. Mladenov, M. de Leon, Softex, Sofia, 2006, 154-175. 
[59] M. Gürses, "Nonlocal Fordy-Kulish equations on symmetric spaces", Phys. Lett. A, 381:21 (2017), 1791-1794, arXiv: 1702.03731.

[60] M. J. Ablowitz, X.-D. Luo, Z.H. Musslimani, Inverse scattering transform for the nonlocal nonlinear Schrödinger equation with nonzero boundary conditions, arXiv: 1612.02726.

[61] M. Li, T. Xu, "Dark and antidark soliton interactions in the nonlocal nonlinear Schrödinger equation with the self-induced parity-time-symmetric potential", Phys. Rev. E, 91:3 (2015), 033202, 8 pp.

[62] В. С. Герджиков, Г. Г. Граховски, Р. И. Иванов, “ $N$-волновые уравнения с $\mathcal{P} \mathcal{T}$-симметрией", ТМФ, 188:3 (2016), 397-415.

[63] V.S. Gerdjikov, G. G. Grahovski, R. I. Ivanov, "On integrable wave interactions and Lax pairs on symmetric spaces", Wave Motion, 71 (2017), 53-70.

[64] А.Г. Рейман, "Единая гамильтонова система на полиномиальных пучках и структура стационарных задач", Зап. науч. семин. ЛОМИ, 131 (1983), 118-127.

[65] А.Г. Рейман, М.А. Семёнов-Тян-Шанский, “Алгебры токов и нелинейные уравнения в частных производных", Докл. АН СССР, 251:6 (1980), 1310-1314.

[66] V.S. Gerdjikov, N. A. Kostov, T. I. Valchev, " $N$-wave equations with orthogonal algebras: $\mathbb{Z}_{2}$ and $\mathbb{Z}_{2} \times \mathbb{Z}_{2}$ reductions and soliton solutions", SIGMA, 3 (2007), 039, 19 pp. 